\title{
Peristaltic Transport of a Third-Order Nano-Fluid in a Circular Cylindrical Tube with Radiation and Chemical Reaction
}

\author{
Abeer A. Shaaban \\ Department of Mathematics, Faculty of Education, Ain Shams University, Roxy, Cairo, Egypt \\ Department of Management Information Systems, Faculty of Business Administration in Rass, \\ Qassim University, Qassim, KSA
}

\begin{abstract}
Explicit Finite-Difference method was used to obtain the solution of the system of the non-linear ordinary differential equations which obtained from the non-linear partial differential equations. These equations describe the two-dimensional flow of a MHD third-order Nano-fluid with heat and mass transfer in a circular cylindrical tube having two walls that are transversely displaced by an infinite, harmonic traveling wave of large wave length. Accordingly, the solutions of momentum, energy, concentration, and Nano-particles concentration equations were obtained. The numerical formula of the stream function, the velocity, the temperature, the concentration, and the Nano-particles distributions of the problem were illustrated graphically. Effects of some parameters of this problem such as, local nanoparticle Grashofnumber Br, local temperature Grashof number Gr, Darcy number Da, magnetic field parameter M, Eckert number Ec, Dufour number Nd, Brownian motion parameter Nb, Thermophoresis parameter Nt, Prandtl number Pr, radiation parameter Rn, Lewis number Le, Sort number Sr, and Chemical reaction parameter Rc on those formula were discussed. Also, an estimation of the global error for the numerical values of the solutions is calculated by using Zadunaisky technique.
\end{abstract}

Keywords: Third-order nano-Fluid, peristatic Motion, MHD flows, Porous medium, Radiation, Chemical reaction.

\section{Nomenclature}

A Chemical Reaction rate constant

$B$ Local nanoparticle Grashof number, defined by Eq. (38)

$C$ The concentration of the fluid

$C$ The concentration at the centerline $(y=0)$

$C$ : The concentration at the wall $(y=h)$

C: Nanoparticle susceptibility

${ }^{D}$ Darcy number, defined by Eq. (38)

$D$ Brownian diffusion coefficient B

$D$ Thermophoretic diffusion coefficient

$\underline{E}$ Electrical field

$E$ Eckert number, defined by Eq. (38)

$F$ The external force

$G$ Gravitational acceleration

$G$ Local temperature Grashof number,
$R e$

Reynolds number, defined by Eq. (38)

$R n \quad$ Radiation parameter, defined by Eq. (38)

$S \quad$ The dimensionless nanoparticles

$\mathrm{Sr} \quad$ Sort number, defined by Eq. (38)

$t \quad$ The time

$T \quad$ The fluid temperature

$T_{1} \quad$ The temperature at the centerline $(y=0)$

$T_{2} \quad$ The temperature at the wall $(y=h)$

$\underline{V} \quad$ The velocity vector

Greek symbols 


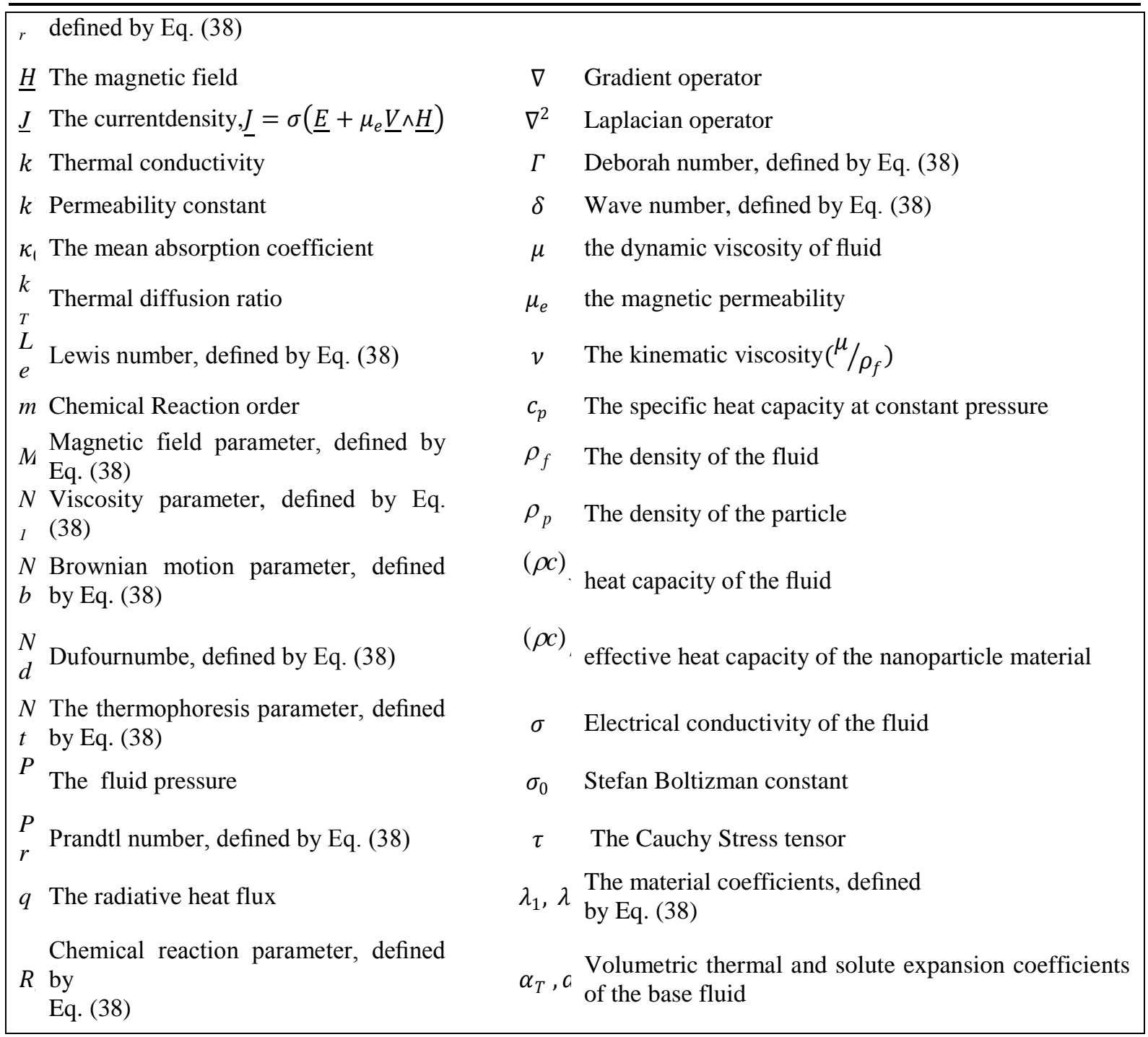

\section{INTRODUCTION}

The analysis of flow dynamics of a fluid in a circular tube induced by a travelling wave on its wall has numerous applications in various branches of science. The word peristaltic stems from the Greek word peristaltikos which means clasping and compressing. The peristaltic transport is a physical mechanism that occurs due to the action of a progressive wave which propagates along thelength of a distensible tube containing fluid. The peristaltic mechanism is nature's way of moving the content within hollow structures by successive contraction on their muscular fibers. This mechanism is responsible for transport of biological fluids such as urine in the ureter, chime in gastro-intestinal tract, semen in the vas deferens and ovum in the female fallopian tube [1]. The application of peristaltic motion as a mean of transporting fluid has aroused interested in engineering fields. Latham [2] was probably the first to study the mechanism of peristaltic pumping in his M. S. Thesis. Several researches have analyzed the phenomenon of peristaltic transport under various assumptions. Haroun [3] studied the effect of a third-order fluid on the peristaltic transport in an asymmetric channel. Eldabe et al. [4] analyzed the incompressible flow of electrically conducting biviscosity fluid through an axisymmetric non-uniform tube with a sinusoidal wave under the considerations of long wavelength and low Reynolds number.

Due to complexity of fluids, several models mainly based on empirical observations have been proposed for non-Newtonian fluids. Amongst these, there is a particular class of fluids, called viscoelastic fluid, which has specially attracted the attention of numerous researchers for varying reasons. The rheologists have been able to provide a theoretical foundation in the form of a constitutive equation which can in principle, have any 'order'. For applied mathematicians and computer scientists the challenge comes from a different quarter. The constitutive equations of even the simplest viscoelastic fluids, namely second grade fluids are such that the differential equations 
describing the motion have, in general, their order higher than those describing the motion of the Newtonian fluids but apparently there is no corresponding increase in the number of boundary conditions. Applied mathematicians and computer scientists are thus forced with the so-called illposed boundary value problems which, in theory, would have a family of infinitely many solutions [5].

Non-Newtonian nanofluids are widely encountered in many industrial and technology applications, for example, melts of polymers, biological solutions, paints, asphalts and glues etc. Nanofluids appear to have the potential to significantly increase heat transfer rates in a variety of areas such as industrial cooling applications, nuclear reactors, transportation industry, micro-electromechanical systems, heat exchangers, chemical catalytic reactors, fiber and granular insulations, packed beds, petroleum reservoirs and nuclear waste repositories and biomedical applications [6].Choi [7] is the first to use the term nano-fluid, who proposed that nanometer sized metallic particles can be suspended in industrial heat transfer fluids. Therefore, a nano-fluid is a suspension of nano-particles (metallic, nonmetallic, or polymeric) in a conventional base fluid which enhances its heat transfer characteristics. Enhanced thermal properties of nano-fluids enable them to use in automotive industry, power plants, cooling systems, computers, etc [8].

In recent years, biomagnetic fluid dynamic is an interesting area of research. This is due to its extensive applications in bioengineering and medical sciences. Examples include the development of magnetic devices for cell separation, targeted transport of drugs using magnetic particles as drug carriers, magnetic wound or cancer tumor treatment causing magnetic hyperthermia, reduction of bleeding during surgeries or provocation of occlusion of the feeding vessels of cancer tumors and development of magnetic tracers [9].

Thermal radiation has a significant role in the overall surface heat transfer when the convection heat transfer coefficient is small. Thermal radiation effect on mixed convection from vertical surface in a porous medium was studied by Bakier[10]. He applied fourth-order Runge-Kutta scheme to solve the governing equations. Effect of MHD flow with mixed convection from radiative vertical isothermal surface embedded in a porous medium was numerically analyzed by Damseh [11]. He used an implicit iterative tri-diagonal finite difference method in order to solve the dimensionless boundarylayer equations. It is well known that the effect of thermal radiation is important in space technology and high temperature processes. Thermal radiation also plays an important role in controlling heat transfer process in polymer processing industry. The effect of radiation on heat transfer problems have been studied by Hossain and Takhar [12]. Zahmatkesh [13] has found that the presence of thermal radiation makes temperature distribution nearly uniform in the vertical sections inside the enclosure and causes the streamlines to be nearly parallel with the vertical walls. Mohsen et al. [14] studied the effect of thermal radiation on magneto-hydrodynamics nanofluid flow and heat transfer by means of two phase model.

Heat and mass transfer problems with chemical reaction are significant in many processes such as drying, evaporations at the surface of a water body, geothermal reservoirs, thermal insulation, enhanced oil recovery, cooling of nuclear reactors and the flow in a desert cooler. This type of flows have many applications in industries. Many practical diffusive operations involve the molecular diffusion of species in the presence of chemical reaction within or at the boundary. Heat and mass transfer effects are also encountered in the chemical industry, in reservoirs, in thermal recovery processes and in the study of hot salty springs in the sea [15]. Vajravelu et al. [16] investigated heat and mass transfer properties of three-layer fluid flow in which nano-fluid layer is squeezed between two clear viscous fluid. Farooq et al. [17] studied heat and mass transfer of two-layer flows of thirdgrade nano-fluids in a vertical channel. Abou-zeid et al. [18] obtained numerical solutions and Global error estimation of natural convection effects on gliding motion of bacteria on a power-law nanoslime through a non-Darcy porous medium. El-Dabe et al. [19] investigated magneto-hydrodynamic non-Newtonian nano-fluid flow over a stretching sheet through a non-Darcy porous medium with radiation and chemical reaction.Shaaban et al. [20] studied the effects of heat and mass transfer on MHD peristaltic flow of a non-Newtonian fluid through a porous medium between two coaxial cylinders.

The objective of this work is to investigate the numerical solution by using Explicit Finite Difference method [21] for the system of non-linear differential equations which arises from the two- 
dimensional flow of a magneto-hydrodynamic third-order Nano-fluid with heat and mass transfer in a circular cylindrical tube having two walls that are transversely displaced by an infinite, harmonic traveling wave of large wave length.We obtained the distributions of the stream function, the velocity, the temperature, the concentration, and the Nanoparticles. Numerical results are found for different values of various non-dimensional parameters. The results are shown graphically and discussed in detail. Also, the global error estimation for the error propagation is obtained by Zadunaisky technique [22].

\section{FLUID MODEL}

By using arguments of modern continuum thermodynamics, the model for a fluid of third grade has been derived by Fosdick and Rajagopal [23]. They show that the constitutive relation for the stress tensor for an incompressible, third-order, homogeneous fluid has the form,

$\bar{\tau}=-\bar{P} I+\bar{S},(1)$

$\bar{S}=\mu \bar{A}_{1}+\alpha_{1} \bar{A}_{2}+\alpha_{2} \bar{A}_{1}^{2}+\beta\left(\operatorname{tr} \bar{A}_{1}^{2}\right) \bar{A}_{1} \quad$.

Here, $-\bar{P} I$ is the indeterminate part of the stress due to the constraint of incompressibility, $\bar{A}_{n}$ are the Rivlin-Ericksen tensors, defined by

$$
\begin{gathered}
\bar{A}_{1}=(\nabla \bar{V})+(\nabla \bar{V})^{T} \\
\bar{A}_{n}=\frac{d \bar{A}_{n-1}}{d \bar{t}}+\bar{A}_{n-1}(\nabla \bar{V})+(\nabla \bar{V})^{T} \bar{A}_{n-1}, \quad n>1 .
\end{gathered}
$$

Where, $\bar{V}$ is the velocity and $\frac{d}{d \bar{t}}$ is the material time derivative. The clausius-Duhem inequality and the requirement that the free energy be a minimum in equilibrium imposes the following constraints on the dynamic viscosity $\mu$, the normal stress coefficients $\alpha_{1}$ and $\alpha_{2}$, and the coefficient $\beta$,

$\mu \geq 0, \alpha_{1} \geq 0, \beta \geq 0,\left|\alpha_{1}+\alpha_{2}\right| \leq \sqrt{24 \mu \beta}$.

Further remarks of third-order fluid models may be found in Dunn and Rajagopal [24].

\section{Mathematical Formulation}

Consider a two-dimensional infinite tube of uniform width $2 n$ filled with an incompressible nonNewtonian nano-fluid obeying third-order model through a porous medium with heat and mass transfer of solar radiation with chemical reaction. A uniform magnetic field intensity $H_{0}$ is imposed and acting along the $\bar{Y}$-axis. By using cartesian coordinates, the tube walls are parallel to the $\bar{X}$-axis and located at $\bar{Y}= \pm n$.

We assume that an infinite train of sinusoidal waves progresses with velocity $\mathrm{c}$ along the walls in the $\bar{X}-$ direction.

The geometry of the wall surface is defined as

$\bar{h}(\bar{x}, \bar{t})=n+b \sin \left(\frac{2 \pi}{\lambda}(\bar{x}-c \bar{t})\right) \quad$.

Where, $b$ is the amplitude and $\lambda$ is the wavelength. We also assume that there is no motion of the wall in the longitudinal direction.

\section{BASIC EqUATIONS}

The basic equations governing the flow of an incompressible Nano-fluid are the following equations $[25,26]$,

The continuity equation

$\nabla \cdot \underline{V}=0$,

The momentum equation

$\rho_{f} \frac{d \bar{V}}{d \bar{t}}=\nabla \cdot \bar{\tau}+\mu_{e}(\underline{J} \wedge \underline{H})-\frac{\mu}{k^{*}} \underline{\bar{V}}+\underline{F},(7)$ 
The energy equation

$$
\begin{aligned}
\left(\rho c_{p}\right)_{f}\left[\frac{d T}{d \bar{t}}\right]=\kappa & \nabla^{2} T+\Phi+\nabla \cdot q+\left(\rho c_{p}\right)_{p}\left[D_{B}(\nabla T \cdot \nabla \hat{\phi})+\frac{D_{T}}{T_{1}}(\nabla T)^{2}\right] \\
& +\frac{\rho_{f} D_{B} \kappa_{T}}{C_{s}} \nabla^{2} C,(8)
\end{aligned}
$$

The concentration equation

$\frac{d C}{d \bar{t}}=D_{B} \nabla^{2} C+\frac{D_{T} \kappa_{T}}{T_{1}} \nabla^{2} T-A\left(C-C_{2}\right)^{m}$,

The nanoparticles concentration equation

$\frac{d \widehat{\phi}}{d \bar{t}}=D_{B} \nabla^{2} \hat{\phi}+\frac{D_{T}}{T_{2}} \nabla^{2} T$

For unsteady two-dimensional flows, velocity components can be written as follows,

$\bar{V}=(\bar{U}(\bar{X}, \bar{Y}, \bar{t}), \bar{V}(\bar{X}, \bar{Y}, \bar{t}), 0)$

Also, the temperature, the concentration, and the nanoparticles functions can be written as follows,

$T=T(\bar{X}, \bar{Y}, \bar{t}) \quad, \quad C=C(\bar{X}, \bar{Y}, \bar{t}), \quad \hat{\phi}(\bar{X}, \bar{Y}, \bar{t})$.

The equations (6), (7) and the constitutive relations (1), (2), and (3) take the following form:-

$\frac{\partial \bar{U}}{\partial \bar{X}}+\frac{\partial \bar{V}}{\partial \bar{Y}}=0$

$\rho_{f}\left(\frac{\partial}{\partial \bar{t}}+\bar{U} \frac{\partial}{\partial \bar{X}}+\bar{V} \frac{\partial}{\partial \bar{Y}}\right) \bar{U}=-\frac{\partial \bar{P}}{\partial \bar{X}}+\mu\left(\frac{\partial^{2}}{\partial \bar{X}^{2}}+\frac{\partial^{2}}{\partial \bar{Y}^{2}}\right) \bar{U}-\frac{\mu}{k^{*}} \bar{U}-\sigma \mu_{e}^{2} H_{0}^{2} \bar{U}+\frac{\partial \bar{S}_{\overline{X X}}}{\partial \bar{X}}+\frac{\partial \bar{S}_{\overline{X Y}}}{\partial \bar{Y}}+$ $\rho_{f} g \alpha_{T}\left(T-T_{2}\right)+\rho_{f} g \alpha_{C}\left(C-C_{2}\right)$,

$\rho_{f}\left(\frac{\partial}{\partial \bar{t}}+\bar{U} \frac{\partial}{\partial \bar{X}}+\bar{V} \frac{\partial}{\partial \bar{Y}}\right) \bar{V}=-\frac{\partial \bar{P}}{\partial \bar{Y}}+\mu\left(\frac{\partial^{2}}{\partial \bar{X}^{2}}+\frac{\partial^{2}}{\partial \bar{Y}^{2}}\right) \bar{V}-\frac{\mu}{k^{*}} \bar{V}+\frac{\partial \bar{S}_{\overline{X Y}}}{\partial \bar{X}}+\frac{\partial \bar{S}_{\overline{Y Y}}}{\partial \bar{Y}}$,

$\bar{S}_{\overline{X X}}=$

$$
\begin{gathered}
2 \mu \frac{\partial \bar{U}}{\partial \bar{X}}+4\left(\alpha_{1}+\alpha_{2}\right)\left(\frac{\partial \bar{U}}{\partial \bar{X}}\right)^{2}+2 \alpha_{1} \frac{\partial \bar{U}}{\partial \bar{Y}}\left(\frac{\partial \bar{V}}{\partial \bar{X}}+\frac{\partial \bar{U}}{\partial \bar{Y}}\right)+\alpha_{2}\left(\frac{\partial \bar{V}}{\partial \bar{X}}+\frac{\partial \bar{U}}{\partial \bar{Y}}\right)^{2}+ \\
2 \beta \frac{\partial \bar{U}}{\partial \bar{X}}\left(4\left(\frac{\partial \bar{U}}{\partial \bar{X}}\right)^{2}+4\left(\frac{\partial \bar{V}}{\partial \bar{Y}}\right)^{2}+2\left(\frac{\partial \bar{V}}{\partial \bar{X}}+\frac{\partial \bar{U}}{\partial \bar{Y}}\right)^{2}\right), \\
\bar{S}_{\overline{X Y}}=\bar{S}_{\overline{Y X}}=\mu\left(\frac{\partial \bar{V}}{\partial \bar{X}}+\frac{\partial \bar{U}}{\partial \bar{Y}}\right)+2 \alpha_{1}\left(\frac{\partial \bar{U}}{\partial \bar{X}} \frac{\partial \bar{V}}{\partial \bar{X}}+\frac{\partial \bar{U}}{\partial \bar{Y}} \frac{\partial \bar{V}}{\partial \bar{Y}}\right)+\left(\alpha_{1}+2 \alpha_{2}\right)\left(\frac{\partial \bar{U}}{\partial \bar{X}}+\frac{\partial \bar{V}}{\partial \bar{Y}}\right) \\
\left(\frac{\partial \bar{V}}{\partial \bar{X}}+\frac{\partial \bar{U}}{\partial \bar{Y}}\right)+\beta\left(4\left(\frac{\partial \bar{U}}{\partial \bar{X}}\right)^{2}+4\left(\frac{\partial \bar{V}}{\partial \bar{Y}}\right)^{2}+2\left(\frac{\partial \bar{V}}{\partial \bar{X}}+\frac{\partial \bar{U}}{\partial \bar{Y}}\right)^{2}\right)\left(\frac{\partial \bar{V}}{\partial \bar{X}}+\frac{\partial \bar{U}}{\partial \bar{Y}}\right),
\end{gathered}
$$

$\bar{S}_{\overline{Y Y}}=$

$$
\begin{array}{r}
2 \mu \frac{\partial \bar{V}}{\partial \bar{Y}}+2 \alpha_{1} \frac{\partial \bar{V}}{\partial \bar{X}}\left(\frac{\partial \bar{V}}{\partial \bar{X}}+\frac{\partial \bar{U}}{\partial \bar{Y}}\right)+4\left(\alpha_{1}+\alpha_{2}\right)\left(\frac{\partial \bar{V}}{\partial \bar{Y}}\right)^{2}+\alpha_{2}\left(\frac{\partial \bar{V}}{\partial \bar{X}}+\frac{\partial \bar{U}}{\partial \bar{Y}}\right)^{2}+ \\
2 \beta \frac{\partial \bar{V}}{\partial \bar{Y}}\left(4\left(\frac{\partial \bar{U}}{\partial \bar{X}}\right)^{2}+4\left(\frac{\partial \bar{V}}{\partial \bar{Y}}\right)^{2}+2\left(\frac{\partial \bar{V}}{\partial \bar{X}}+\frac{\partial \bar{U}}{\partial \bar{Y}}\right)^{2}\right) .
\end{array}
$$

The dissipation function $\Phi$ can be written as follows

$\Phi=\bar{\tau}_{i j} \frac{\partial \bar{V}_{i}}{\partial \bar{X}_{j}}$

$\Phi=\bar{S}_{\overline{X X}} \frac{\partial \bar{U}}{\partial \bar{X}}+\bar{S}_{\overline{X Y}}\left(\frac{\partial \bar{V}}{\partial \bar{X}}+\frac{\partial \bar{U}}{\partial \bar{Y}}\right)+\bar{S}_{\overline{Y Y}} \frac{\partial \bar{V}}{\partial \bar{Y}} \quad$,

Also, by using Rosselant approximation [27] we have,

$q=\frac{4 \sigma_{0}}{3 \kappa_{0}} \frac{\partial T^{4}}{\partial Y}$, 
We assume that the temperature differences within the flow are sufficiently small such that $T^{4}$ may be expressed as a linear function of temperature. This is accomplished by expanding $T^{4}$ in a Taylor series about $T_{2}$, and neglecting higher-order terms [28], one gets,

$T^{4} \cong 4 T_{2}^{3} T-3 T_{2}^{4}$

Then, equations (8), (9), and (10) can be written as follows:-

$$
\begin{aligned}
& \left(\rho c_{p}\right)_{f}\left[\frac{\partial T}{\partial \bar{t}}+\bar{U} \frac{\partial T}{\partial \bar{X}}+\bar{V} \frac{\partial T}{\partial \bar{Y}}\right]=\kappa\left(\frac{\partial^{2} T}{\partial \bar{X}^{2}}+\frac{\partial^{2} T}{\partial \bar{Y}^{2}}\right)+\frac{16 \sigma_{0}}{3 \kappa_{0}} T_{2}^{3} \frac{\partial^{2} T}{\partial \bar{Y}^{2}}+\bar{S}_{\overline{X X}} \frac{\partial \bar{U}}{\partial \bar{X}}+\bar{S}_{\overline{X Y}}\left(\frac{\partial \bar{V}}{\partial \bar{X}}+\frac{\partial \bar{U}}{\partial \bar{Y}}\right)+\bar{S}_{\overline{Y Y}} \frac{\partial \bar{V}}{\partial \bar{Y}}+ \\
& \left(\rho c_{p}\right)_{p}\left[D_{B}\left(\frac{\partial T}{\partial \bar{X}} \cdot \frac{\partial \widehat{\phi}}{\partial \bar{X}}+\frac{\partial T}{\partial \bar{Y}} \frac{\partial \widehat{\phi}}{\partial \bar{Y}}\right)+\frac{D_{T}}{T_{1}}\left(\left(\frac{\partial T}{\partial \bar{X}}\right)^{2}+\left(\frac{\partial T}{\partial \bar{Y}}\right)^{2}\right)\right]+\frac{\rho_{f} D_{B} \kappa_{T}}{C_{s}}\left(\frac{\partial^{2} C}{\partial \bar{X}^{2}}+\frac{\partial^{2} C}{\partial \bar{Y}^{2}}\right), \\
& {\left[\frac{\partial C}{\partial \bar{t}}+\bar{U} \frac{\partial C}{\partial \bar{X}}+\bar{V} \frac{\partial C}{\partial \bar{Y}}\right]=D_{B}\left(\frac{\partial^{2} C}{\partial \bar{X}^{2}}+\frac{\partial^{2} C}{\partial \bar{Y}^{2}}\right)+\frac{D_{T} \kappa_{T}}{T_{1}}\left(\frac{\partial^{2} T}{\partial \bar{X}^{2}}+\frac{\partial^{2} T}{\partial \bar{Y}^{2}}\right)-A\left(C-C_{2}\right)^{m},} \\
& {\left[\frac{\partial \widehat{\phi}}{\partial \bar{t}}+\bar{U} \frac{\partial \widehat{\phi}}{\partial \bar{X}}+\bar{V} \frac{\partial \widehat{\phi}}{\partial \bar{Y}}\right]=D_{B}\left(\frac{\partial^{2} \widehat{\phi}}{\partial \bar{X}^{2}}+\frac{\partial^{2} \widehat{\phi}}{\partial \bar{Y}^{2}}\right)+\frac{D_{T}}{T_{2}}\left(\frac{\partial^{2} T}{\partial \bar{X}^{2}}+\frac{\partial^{2} T}{\partial \bar{Y}^{2}}\right)}
\end{aligned}
$$

In the fixed coordinate system $(\bar{X}, \bar{Y})$, the motion is unsteady because of the moving boundary. However, if observed in a coordinate system $(\bar{x}, \bar{y})$ moving with the speed $c$, it can be treated as steady because the boundary shape appears to be stationary.

The transformation between the two frames is given by

$\bar{x}=\bar{X}-c \bar{t} \quad \bar{y}=\bar{Y}$.

The velocities in the fixed and moving frames are related by

$\bar{u}=\bar{U}-c \quad \bar{v}=\bar{V}$.

Where, $\bar{u}, \bar{v}$ are components of the velocity in the moving coordinate system.

And, in order to simplify the governing equations of the motion, we may introduce the following dimensionless transformations:-

$$
\begin{gathered}
x=\frac{2 \pi}{\lambda} \bar{x} \quad, \quad y=\frac{\bar{y}}{n}, \quad u=\frac{\bar{u}}{c} \quad, \quad v=\frac{\bar{v}}{c}, \\
s=\frac{n}{\pi c} \bar{s} \quad, \quad p=\frac{2 \pi n^{2}}{\lambda(\mu+\eta) c} \bar{p} \quad, \quad h=\frac{\bar{h}}{n}, \\
\theta=\frac{T-T_{2}}{T_{1}-T_{2}} \quad, \quad \phi=\frac{C-C_{2}}{C_{1}-C_{2}} \quad, \quad S=\frac{\widehat{\phi}-\widehat{\phi}_{2}}{\widehat{\phi}_{1}-\widehat{\phi}_{2}} .
\end{gathered}
$$

Substituting by equations (26), (27), and (28) into equations (13) - (18), and (23) - (25) we obtain the following non-dimensionless equations:-

$$
\begin{aligned}
& \delta \frac{\partial u}{\partial x}+\frac{\partial v}{\partial y}=0 \text {, } \\
& \operatorname{Re}\left(\delta u \frac{\partial u}{\partial x}+v \frac{\partial u}{\partial y}\right)=-\left(1+N_{1}\right) \frac{\partial p}{\partial x}+\left(\delta^{2} \frac{\partial^{2} u}{\partial x^{2}}+\frac{\partial^{2} u}{\partial y^{2}}\right)-\frac{1}{D a} u-M u+\delta \frac{\partial s_{x x}}{\partial x}+\frac{\partial s_{x y}}{\partial y}+ \\
& \operatorname{Gr} \theta+B r \phi \text {, } \\
& \delta \operatorname{Re}\left(\delta u \frac{\partial v}{\partial x}+v \frac{\partial v}{\partial y}\right)=-\left(1+N_{1}\right) \frac{\partial p}{\partial y}+\delta\left(\delta^{2} \frac{\partial^{2} v}{\partial x^{2}}+\frac{\partial^{2} v}{\partial y^{2}}\right)-\frac{\delta}{D a} v+\delta^{2} \frac{\partial s_{x y}}{\partial x}+\delta \frac{\partial s_{y y}}{\partial y}, \\
& s_{x x}= \\
& 2 \delta \frac{\partial u}{\partial x}+4\left(\lambda_{1}+\lambda_{2}\right) \delta^{2}\left(\frac{\partial u}{\partial x}\right)^{2}+2 \lambda_{1} \frac{\partial u}{\partial y}\left(\delta \frac{\partial v}{\partial x}+\frac{\partial u}{\partial y}\right)+\lambda_{2}\left(\delta \frac{\partial v}{\partial x}+\frac{\partial u}{\partial y}\right)^{2}+ \\
& 2 \Gamma \delta \frac{\partial u}{\partial x}\left(4 \delta^{2}\left(\frac{\partial u}{\partial x}\right)^{2}+4\left(\frac{\partial v}{\partial y}\right)^{2}+2\left(\delta \frac{\partial v}{\partial x}+\frac{\partial u}{\partial y}\right)^{2}\right), \\
& s_{x y}=s_{y x}=\left(\delta \frac{\partial v}{\partial x}+\frac{\partial u}{\partial y}\right)+2 \lambda_{1}\left(\delta^{2} \frac{\partial u}{\partial x} \frac{\partial v}{\partial x}+\frac{\partial u}{\partial y} \frac{\partial v}{\partial y}\right)+\left(\lambda_{1}+2 \lambda_{2}\right)\left(\delta \frac{\partial u}{\partial x}+\frac{\partial v}{\partial y}\right)
\end{aligned}
$$




$$
\begin{gathered}
\left(\delta \frac{\partial v}{\partial x}+\frac{\partial u}{\partial y}\right)+\Gamma\left(4 \delta^{2}\left(\frac{\partial u}{\partial x}\right)^{2}+4\left(\frac{\partial v}{\partial y}\right)^{2}+2\left(\delta \frac{\partial v}{\partial x}+\frac{\partial u}{\partial y}\right)^{2}\right)\left(\delta \frac{\partial v}{\partial x}+\frac{\partial u}{\partial y}\right),(33) \\
s_{y y}=2 \frac{\partial v}{\partial y}+2 \lambda_{1} \delta \frac{\partial v}{\partial x}\left(\delta \frac{\partial v}{\partial x}+\frac{\partial u}{\partial y}\right)+4\left(\lambda_{1}+\lambda_{2}\right)\left(\frac{\partial v}{\partial y}\right)^{2}+ \\
\lambda_{2}\left(\delta \frac{\partial v}{\partial x}+\frac{\partial u}{\partial y}\right)^{2}+2 \Gamma \frac{\partial v}{\partial y}\left(4 \delta^{2}\left(\frac{\partial u}{\partial x}\right)^{2}+4\left(\frac{\partial v}{\partial y}\right)^{2}+2\left(\delta \frac{\partial v}{\partial x}+\frac{\partial u}{\partial y}\right)^{2}\right) .
\end{gathered}
$$

$R e\left[\delta u \frac{\partial \theta}{\partial x}+v \frac{\partial \theta}{\partial y}\right]=\frac{1}{P r}\left(\delta^{2} \frac{\partial^{2} \theta}{\partial x^{2}}+\frac{\partial^{2} \theta}{\partial y^{2}}\right)+\frac{4}{3 R n} \frac{\partial^{2} \theta}{\partial y^{2}}+E c \delta s_{x x} \frac{\partial u}{\partial x}+E c s_{x y}\left(\delta \frac{\partial v}{\partial x}+\frac{\partial u}{\partial y}\right)+E c s_{y y} \frac{\partial v}{\partial y}+$

$\left[N b\left(\delta^{2} \frac{\partial \theta}{\partial x} \cdot \frac{\partial S}{\partial x}+\frac{\partial \theta}{\partial y} \cdot \frac{\partial S}{\partial y}\right)+N t\left(\delta^{2}\left(\frac{\partial \theta}{\partial x}\right)^{2}+\left(\frac{\partial \theta}{\partial y}\right)^{2}\right)\right]+N d\left(\delta^{2} \frac{\partial^{2} \phi}{\partial x^{2}}+\frac{\partial^{2} \phi}{\partial y^{2}}\right)$,

$\operatorname{Re}\left[\delta u \frac{\partial \phi}{\partial x}+v \frac{\partial \phi}{\partial y}\right]=\frac{1}{L e}\left(\delta^{2} \frac{\partial^{2} \phi}{\partial x^{2}}+\frac{\partial^{2} \phi}{\partial y^{2}}\right)+\operatorname{Sr}\left(\delta^{2} \frac{\partial^{2} \theta}{\partial x^{2}}+\frac{\partial^{2} \theta}{\partial y^{2}}\right)-R c(\phi)^{m}$,

$R e\left[\delta u \frac{\partial S}{\partial x}+v \frac{\partial S}{\partial y}\right]=N b\left(\delta^{2} \frac{\partial^{2} S}{\partial x^{2}}+\frac{\partial^{2} S}{\partial y^{2}}\right)+N t\left(\delta^{2} \frac{\partial^{2} \theta}{\partial x^{2}}+\frac{\partial^{2} \theta}{\partial y^{2}}\right)$

Where, the dimensionless parameters are defined by:-

$$
\begin{aligned}
& \delta=\frac{2 \pi n}{\lambda}, \quad R e=\frac{\rho c n}{\mu}, N_{1}=\frac{\eta}{\mu}, D a=\frac{k^{*}}{n^{2}}, M=\frac{\sigma \mu_{e}^{2} H_{0}^{2} n^{2}}{\mu} \\
& \lambda_{1}=\frac{\alpha_{1} c}{\mu n} \quad, \quad \lambda_{2}=\frac{\alpha_{2} c}{\mu n}, \Gamma=\frac{\beta c^{2}}{\mu n^{2}}, G r=\frac{g n \alpha_{T}\left(T_{1}-T_{2}\right)}{c^{2}}, B r=\frac{g n \alpha_{C}\left(C_{1}-C_{2}\right)}{c^{2}} \\
& \operatorname{Pr}=\frac{v\left(\rho c_{p}\right)_{f}}{\kappa}, R n=\frac{\left(\rho c_{p}\right)_{f} \kappa_{0} v}{4 \sigma_{0} T_{2}^{3}}, E c=\frac{c^{2}}{c_{p}\left(T_{1}-T_{2}\right)}, N d=\frac{D_{B} \kappa_{T}\left(C_{1}-C_{2}\right)}{C_{s} c_{p} v\left(T_{1}-T_{2}\right)} \quad, N b=\frac{D_{B}\left(\widehat{\phi}_{1}-\widehat{\phi}_{2}\right)}{v} \\
& N t=\frac{D_{T}\left(T_{1}-T_{2}\right)}{T_{1} v} \quad, \quad L e=\frac{v}{D_{B}} \quad, \quad S r=\frac{D_{T} \kappa_{T}\left(T_{1}-T_{2}\right)}{T_{1} v\left(C_{1}-C_{2}\right)}, \quad R_{C}=\frac{n^{2} A\left(C_{1}-C_{2}\right)^{m-1}}{v}
\end{aligned}
$$

Equation (29) allows the introducing of the dimensionless stream function $\psi(x, y)$ in terms of

$u=\frac{\partial \psi}{\partial y} \quad, \quad v=-\delta \frac{\partial \psi}{\partial x} \quad$.

Then, we carry out our investigation on the basis that the dimensionless wave number is small, that is,

$\delta<<1$,

which corresponds to the long-wavelength approximation [29]. Thus, to lowest order in $\delta$, equations $(30-37)$ give,

$\left(1+N_{1}\right) \frac{\partial p}{\partial x}=\left(\frac{\partial^{3} \psi}{\partial y^{3}}\right)-\left(\frac{1}{D a}+M\right) \frac{\partial \psi}{\partial y}+\frac{\partial s_{x y}}{\partial y}+\operatorname{Gr} \theta+\operatorname{Br} \phi$,

$\frac{\partial p}{\partial y}=0$

$s_{x x}=\left(2 \lambda_{1}+\lambda_{2}\right)\left(\frac{\partial^{2} \psi}{\partial y^{2}}\right)^{2}$

$s_{x y}=s_{y x}=\left(\frac{\partial^{2} \psi}{\partial y^{2}}\right)+2 \Gamma\left(\frac{\partial^{2} \psi}{\partial y^{2}}\right)^{3}$

$s_{y y}=\lambda_{2}\left(\frac{\partial^{2} \psi}{\partial y^{2}}\right)^{2}$

$\left(\frac{1}{P r}+\frac{4}{3 R n}\right) \frac{\partial^{2} \theta}{\partial y^{2}}+E c S_{x y}\left(\frac{\partial^{2} \psi}{\partial y^{2}}\right)+N b\left(\frac{\partial \theta}{\partial y} \cdot \frac{\partial S}{\partial y}\right)+N t\left(\frac{\partial \theta}{\partial y}\right)^{2}+N d\left(\frac{\partial^{2} \phi}{\partial y^{2}}\right)=0 \quad$,

$\frac{1}{L e}\left(\frac{\partial^{2} \phi}{\partial y^{2}}\right)+\operatorname{Sr}\left(\frac{\partial^{2} \theta}{\partial y^{2}}\right)-R c(\phi)^{m}=0 \quad$, 
$N b\left(\frac{\partial^{2} S}{\partial y^{2}}\right)+N t\left(\frac{\partial^{2} \theta}{\partial y^{2}}\right)=0$

\section{Boundary conditions in dimensionless form are:-}

For the stream function in the moving frame are,

$\left.\begin{array}{rl}\psi & =0 \quad, \quad \text { (by convection) } \\ \frac{\partial^{2} \psi}{\partial y^{2}} & =0 \quad, \quad(\text { by symmetry })\end{array}\right\}$ on the centerline $y=0$,

$\left.\begin{array}{l}\left.\frac{\partial \psi}{\partial y}=-1 \quad, \quad \text { (no slip condition }\right) \\ \psi=F\end{array}\right\}$

at the wall $y=h$.

Where, $F$ is the total flux number. We also note that hrepresents the dimensionless form of the surface of the peristaltic wall.

$h(x)=1+\chi \sin x$.

Where, $\chi=\frac{b}{n}$ (is the amplitude ratio or the occlusion)

For the temperature, the concentration, and the nanoparticles are,

$$
\theta=1, \quad \phi=1, \quad S=1, \quad \text { at } y=0,
$$

$\theta=0, \quad \phi=0, \quad S=0, \quad$ at $y=h$.

Then the dimensionless boundary conditions can be written as

$$
\begin{gathered}
\psi=0, \frac{\partial^{2} \psi}{\partial y^{2}}=0, \theta=1, \quad \phi=1, \quad S=1, \quad \text { at } y=0, \\
\psi=F, \frac{\partial \psi}{\partial y}=-1, \theta=0, \phi=0, \quad S=0, \quad \text { at } y=h .
\end{gathered}
$$

Substituting (44) into (41) and (46) and using (42) we finally have,

$$
\begin{aligned}
& \psi^{(4)}=\frac{\left(\frac{1}{D a}+M\right) \psi^{\prime \prime}-G r \theta^{\prime}-B r \phi^{\prime}-12 \Gamma \psi^{\prime \prime} \psi^{\prime \prime \prime} 2}{\left(2+6 \Gamma \psi^{\prime 2}\right)}, \\
& \theta^{\prime \prime}=\frac{-E c \psi^{\prime \prime 2}-2 E c \Gamma \psi^{\prime \prime}-N d \phi^{\prime \prime}-N b \theta^{\prime} s^{\prime}-N t \theta^{2}}{\left(\frac{1}{P r}+\frac{4}{3 R n}\right)},
\end{aligned}
$$

$\phi^{\prime \prime}=-\operatorname{Le} \operatorname{Sr} \theta^{\prime \prime}+\operatorname{Le} \operatorname{Rc} \phi^{m}$,

$S^{\prime \prime}=-\frac{N t}{N b} \theta^{\prime \prime}$

The system of non-linear ordinary differential (52) together with the boundary conditions (51), will be solved numerically by using the explicit finite-difference method. And, we computed the global error for the solutions of the problem.

\section{NUMERICAL SOLUTION}

The equations (52) can be written after applied explicit finite difference schemes [21] as:

$\left(\frac{2 \psi[i+2]-9 \psi[i+1]+16 \psi[i]-14 \psi[i-1]+6 \psi[i-2]-\psi[i-3]}{h^{4}}\right)\left(2+6 \Gamma\left(\frac{\psi[i+1]-2 \psi[i]+\psi[i-1]}{h^{2}}\right)^{2}\right)-[(M+$

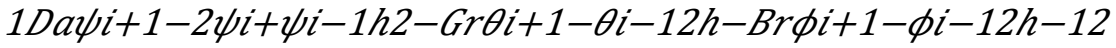

$\Gamma \psi i+1-2 \psi i+\psi i-1 h 2 \psi i+2-3 \psi i+1+3 \psi i-\psi i-1 h 32=0$,

$\left(\frac{1}{P r}+\frac{4}{3 R n}\right)\left(\frac{\theta[i+1]-2 \theta[i]+\theta[i-1]}{h^{2}}\right)+E c\left(\frac{\psi[i+1]-2 \psi[i]+\psi[i-1]}{h^{2}}\right)^{2}+2 E c \Gamma\left(\frac{\psi[i+1]-2 \psi[i]+\psi[i-1]}{h^{2}}\right)^{4}+$ $N d\left(\frac{\phi[i+1]-2 \phi[i]+\phi[i-1]}{h^{2}}\right)+N b\left(\frac{\theta[i+1]-\theta[i-1]}{2 h}\right)\left(\frac{S[i+1]-S[i-1]}{2 h}\right)+N t\left(\frac{\theta[i+1]-\theta[i-1]}{2 h}\right)^{2}=0 \quad$, 


$$
\begin{aligned}
& \left(\frac{\phi[i+1]-2 \phi[i]+\phi[i-1]}{h^{2}}\right)+\operatorname{LeSr}\left(\frac{\theta[i+1]-2 \theta[i]+\theta[i-1]}{h^{2}}\right)-\operatorname{Le} R c(\phi[i])^{m}=0, \\
& \left(\frac{S[i+1]-2 S[i]+S[i-1]}{h^{2}}\right)+\frac{N t}{N b}\left(\frac{\theta[i+1]-2 \theta[i]+\theta[i-1]}{h^{2}}\right)=0,
\end{aligned}
$$

Where the index $i$ refers to $y$ and the $\Delta y=h=0.04$. According to the boundary conditions (51) we can solved equations (53) numerically, then a Newtonian iteration method continues until either of goals specified by accuracy goal or precision goal is achieved.

\section{ESTIMATION OF THE GLOBAL ERROR}

We used Zadunaisky technique [22] for calculating the global error for the solutions of the problem. We calculate an estimation of the global error from the formulas,

$\underline{e}_{n}=\underline{f_{n}}-\underline{f}\left(x_{n}\right)=\underline{f_{n}}-\underline{P}\left(x_{n}\right), \quad(n=0,1,2, \ldots \ldots \ldots, 25)$.

In this relation, $\underline{f}_{n}$ is the approximate solutions of the new problem (the pseudo-problem) at the point $x_{n}$, and $\underline{f}\left(x_{n}\right)$ is the exact solutions of pseudo-problem at $x_{n}$.

The values of global error for the solutions of the problem which solved by the explicit finite difference method are shown in ( tab.1 ). The error in (tab. 1) based on using 26 points to find interpolating polynomials $P_{i}(x)$ of degree 25 . In order to achieve the above task, we used the Mathematica package.

\begin{tabular}{|c|c|c|c|c|c|c|c|c|}
\hline$y$ & $\mathrm{u}=\mathrm{Y} 2$ & error $(e 2 n)$ & $\theta=Y 5$ & error (e5n) & $\phi=Y 7$ & error $\left(e_{7 n}\right)$ & $S=Y 9$ & error (e9n) \\
\hline 0 & -2.37728 & 7.29673E-04 & $1.00 \mathrm{E}+00$ & $0.00 \mathrm{E}+00$ & & $0.00 \mathrm{E}+00$ & $1.00 \mathrm{E}+00$ & $0.00 \mathrm{E}+00$ \\
\hline 0.08 & -2.37381 & $2.89037 \mathrm{E}-04$ & 0.942545 & $2.13292 \mathrm{E}-04$ & 0.916916 & $2.47102 \mathrm{E}-05$ & 0.915491 & $2.34465 \mathrm{E}-04$ \\
\hline 0.16 & -2.36041 & $6.39575 \mathrm{E}-04$ & 0.883307 & $4.12111 \mathrm{E}-04$ & 0.834279 & $4.59696 \mathrm{E}-05$ & 0.831339 & $1.37629 \mathrm{E}-03$ \\
\hline 0.24 & -2.33457 & $1.41259 \mathrm{E}-03$ & 0.822206 & $5.87384 \mathrm{E}-04$ & 0.752052 & $6.27746 \mathrm{E}-05$ & 0.747559 & $3.26827 \mathrm{E}-03$ \\
\hline 0.32 & -2.29477 & $1.74905 \mathrm{E}-03$ & 0.759054 & 7.26556E-04 & $6 / 0211$ & 7.38514E-05 & 0.664189 & $5.73609 \mathrm{E}-03$ \\
\hline 0.4 & -2.2394 & $1.62674 \mathrm{E}-03$ & 0.69352 & $8.12272 \mathrm{E}-04$ & 0.588752 & $7.75766 \mathrm{E}-05$ & 0.581296 & $8.58304 \mathrm{E}-03$ \\
\hline 0.48 & -2.16637 & $1.12886 \mathrm{E}-03$ & 0.625087 & $8.24749 \mathrm{E}-04$ & $0.50 / 695$ & 7.22617E-05 & 0.498983 & $1.15732 \mathrm{E}-02$ \\
\hline 0.56 & -2.07304 & $3.78352 \mathrm{E}-04$ & 0.552965 & 7.45725E-04 & 0.421089 & $5.66182 \mathrm{E}-05$ & 0.417407 & $1.4408 \mathrm{E}-02$ \\
\hline 0.64 & -1.95602 & $4.79557 \mathrm{E}-04$ & 0.475984 & 5.64493E-04 & & 3.04824E-05 & 0.336803 & $1.6695 \mathrm{E}-02$ \\
\hline 0.72 & -1.81115 & $1.27505 \mathrm{E}-03$ & 0.392423 & $2.88096 \mathrm{E}-04$ & & 3.95057E-06 & 0.257515 & $1.79035 \mathrm{E}-02$ \\
\hline 0.8 & -1.6333 & $1.80269 \mathrm{E}-03$ & 0.299767 & $4.04455 \mathrm{E}-05$ & & 3.99397E-05 & 0.180047 & $1.73019 \mathrm{E}-02$ \\
\hline 0.88 & -1.41622 & $1.80209 \mathrm{E}-03$ & 0.194348 & 3.0874E-04 & & $6.23851 \mathrm{E}-05$ & 0.10513 & $1.38654 \mathrm{E}-02$ \\
\hline 0.96 & -1.15231 & $9.21908 \mathrm{E}-04$ & 0.0708377 & 2.72493E-04 & & 4.16893E-05 & 0.0338325 & $6.12791 \mathrm{E}-03$ \\
\hline 1 & $-1.00 \mathrm{E}+00$ & $0.00 \mathrm{E}+00$ & $0.00 \mathrm{E}+00$ & $0.00 \mathrm{E}+00$ & $0.00 \mathrm{E}+00$ & $0.00 \mathrm{E}+00$ & $0.00 \mathrm{E}+00$ & $0.00 \mathrm{E}+00$ \\
\hline
\end{tabular}

Table 1. The global error by using finite difference technique

Note: this table contains the values of the dimensionless physical quantities, the velocity $u$, the temperature $\theta$, the concentration $\phi$, and the nano-particle concentration $\mathrm{S}$ at the values of the dimensionless distance $\mathrm{y}$, Also, this table contains the values of the global error e $2 \mathrm{n}$ of $\mathrm{u}$, the global error e $5 \mathrm{n}$ of $\theta$, the global error e $7 \mathrm{n}$ of $\phi$, and the global error e9n of S, by using finite difference technique.

\section{NUMERICAL RESULTS AND DISCUSSION}

In this paper we generalized the problem ofa magneto-hydrodynamic third-order Nano-fluid with heat and mass transfer in a circular cylindrical tube having two walls that are transversely displaced by an infinite, harmonic traveling wave of large wave length. The system of non-linear ordinary differential equations (52) with the boundary conditions (51) was solved numerically by using an explicit finite difference method. The functions $\psi, u, \theta, \phi$, and $S$ are obtained and illustrated graphically as shown in figures (1-17) for different values of the parameters of the problem. We studied all parameters of the problem, but we selected some of them to save space.

Figure (1) shows the distribution of the velocity profile $u$ at different values of local nanoparticle Grashofnumber Br. It is clear that, the velocity increases by increasing local nanoparticle Grashof 
number $\mathrm{Br}$ in the region $0 \leq y \leq 0.45$ and, it returns decrease at $0.45 \leq y \leq 1$. And, we have led to the local temperature Grashof number Greffects on the velocity the same effect of local nanoparticle Grashofnumber $\mathrm{Br}$ on the velocity.

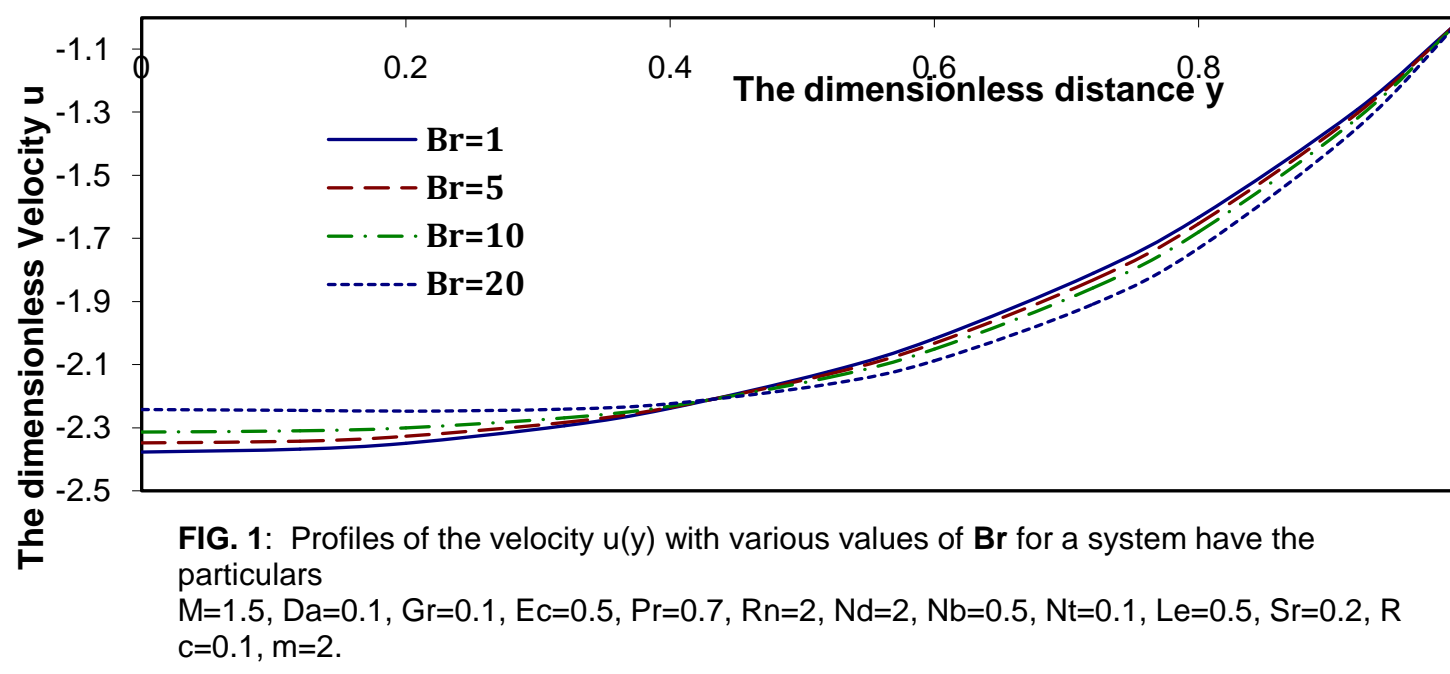

Figure (2) describes the effect of the Darcy number Da on the stream function. It is noted that, by increasing of the Darcy number Da, the stream function decreases. And, we have led to the Darcy number Da effects on the temperature the opposite effect of the Darcy number Da on the stream function. Figure (3) illustrates the distributions of the velocity profile at different values of the Darcy number Da. It is seen that, the velocity decreases by increasing the Darcy number Da in the region $0 \leq y \leq 0.55$ and, it returns increase at $0.55 \leq y \leq 1$.

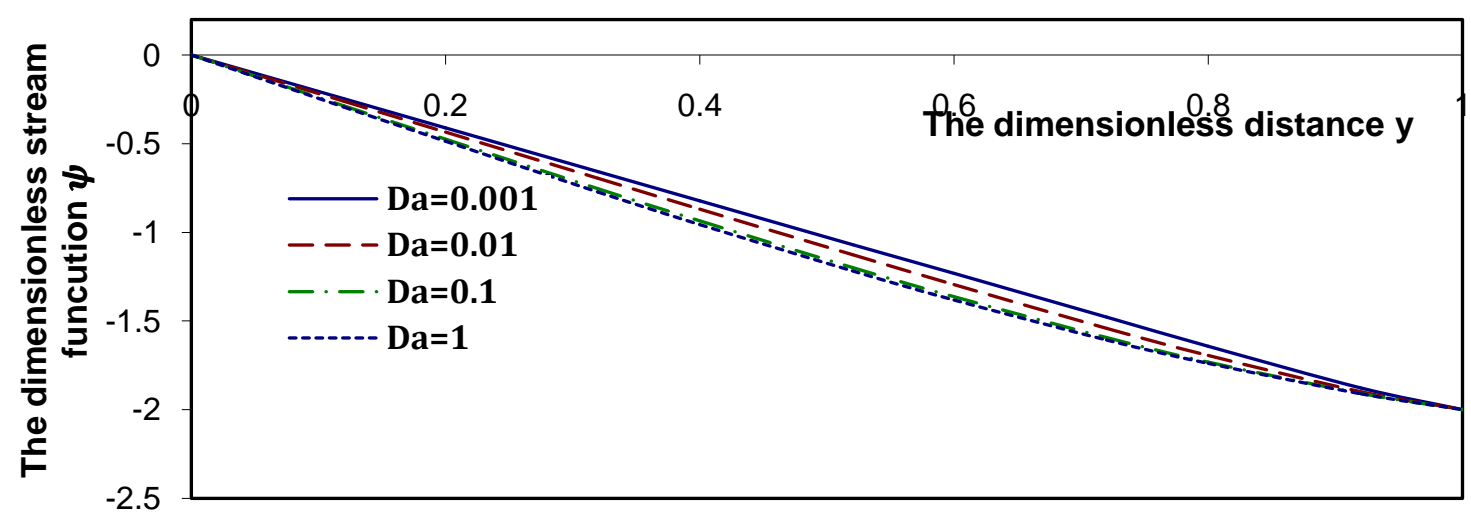

FIG. 2: Profiles of the stream function $\boldsymbol{\psi}(\mathrm{y})$ with various values of $\mathrm{Da}$ for a system have the particulars

$\mathrm{M}=1.5, \mathrm{Gr}=0.1, \mathrm{Br}=0.1, \mathrm{Ec}=0.5, \mathrm{Pr}=0.7, \mathrm{Rn}=2, \mathrm{Nd}=2, \mathrm{Nb}=0.5, \mathrm{Nt}=0.1, \mathrm{Le}=0.5, \mathrm{Sr}=0.2, \mathrm{Rc}$ $=0.1, \mathrm{~m}=2$.

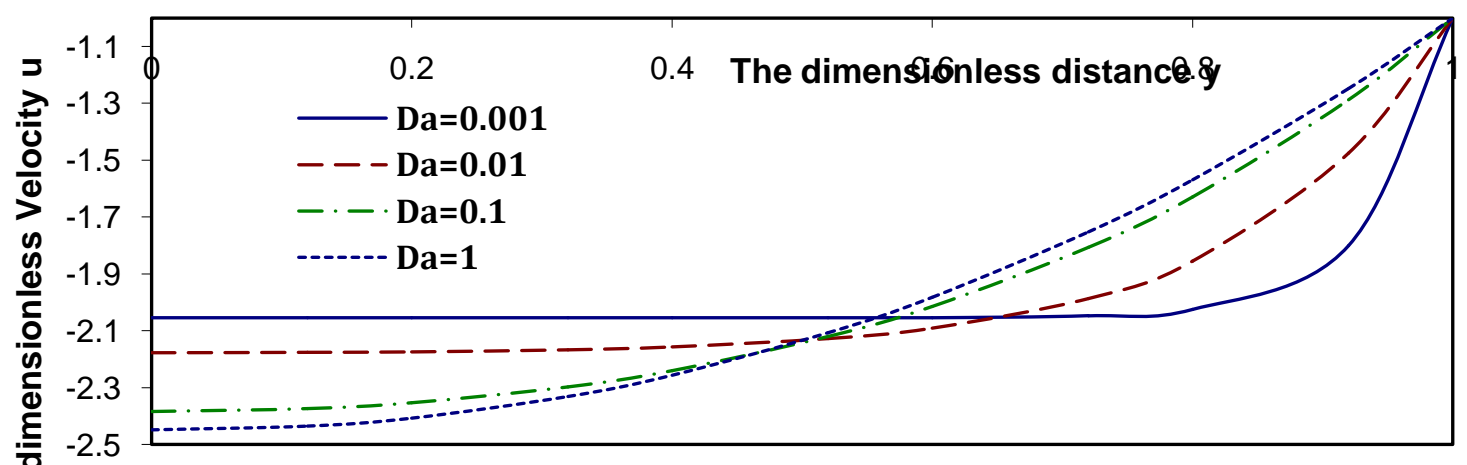

FIG. 3: Profiles of the velocity $u(y)$ with various values of Da for a system have the particulars

$\mathrm{M}=1.5, \mathrm{Gr}=0.1, \mathrm{Br}=0.1, \mathrm{Ec}=0.5, \mathrm{Pr}=0.7, \mathrm{Rn}=2, \mathrm{Nd}=2, \mathrm{Nb}=0.5, \mathrm{Nt}=0.1, \mathrm{Le}=0.5, \mathrm{Sr}=0.2, \mathrm{Rc}$ $=0.1, \mathrm{~m}=2$. 
Peristaltic Transport of a Third-Order Nano-Fluid in a Circular Cylindrical Tube with Radiation and Chemical Reaction

Figure (4) shows the effect of magnetic field parameter $M$ on the stream function. It is clear that, the stream function increases by increasing of magnetic field parameter M. And, we have led to magnetic field parameter $\mathrm{M}$ effects on the temperature the opposite effect of magnetic field parameter $\mathrm{M}$ on the stream function. Figure (5) illustrates the effect of magnetic field parameter $M$ on the velocity profiles. It is clear that, the velocity increases by increasing magnetic field parameter $\mathrm{M}$ in the region $0 \leq y \leq 0.5$ and, it returns decrease at $0.5 \leq y \leq 1$.

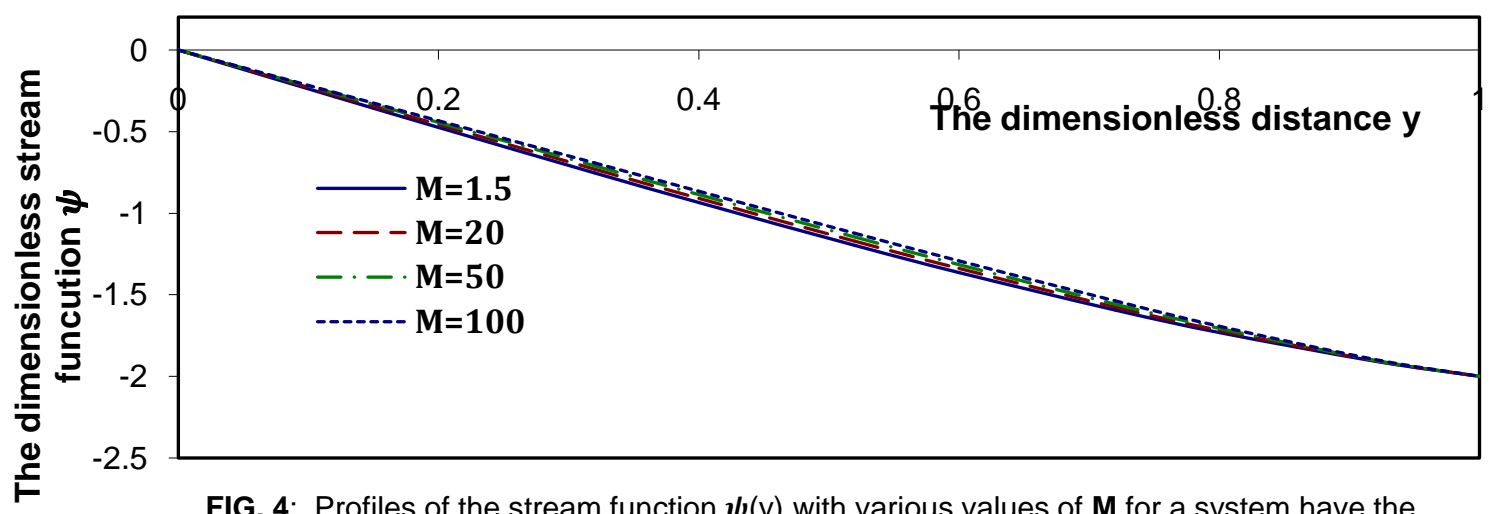

FIG. 4: Profiles of the stream function $\boldsymbol{\psi}(\mathrm{y})$ with various values of $\mathbf{M}$ for a system have the particulars

$\mathrm{Da}=0.1, \mathrm{Gr}=0.1, \mathrm{Br}=0.1, \mathrm{Ec}=0.5, \mathrm{Pr}=0.7, \mathrm{Rn}=2, \mathrm{Nd}=2, \mathrm{Nb}=0.5, \mathrm{Nt}=0.1, \mathrm{Le}=0.5, \mathrm{Sr}=0.2, \mathrm{Rc}=$ $0.1, \mathrm{~m}=2$.

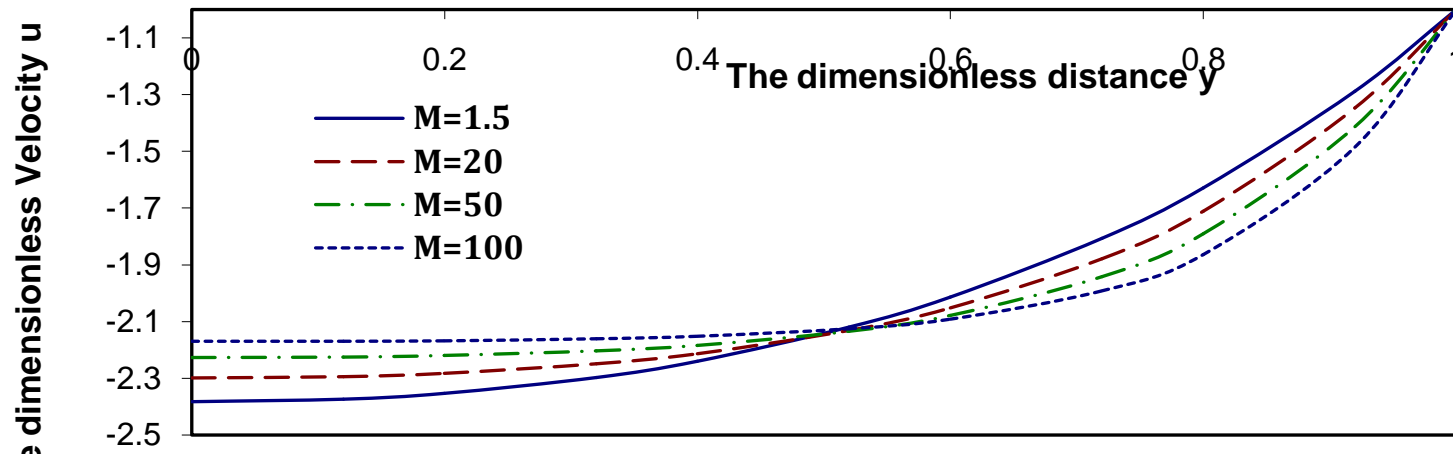

FIG. 5: Profiles of the velocity $\mathrm{u}(\mathrm{y})$ with various values of $\mathbf{M}$ for a system have the particulars $\mathrm{Da}=0.1, \mathrm{Gr}=0.1, \mathrm{Br}=0.1, \mathrm{Ec}=0.5, \mathrm{Pr}=0.7, \mathrm{Rn}=2, \mathrm{Nd}=2, \mathrm{Nb}=0.5, \mathrm{Nt}=0.1$, $\mathrm{Le}=0.5, \mathrm{Sr}=0.2, \mathrm{Rc}=0.1, \mathrm{~m}=2$.

Figure $(6,7)$ show the effect of Eckert number Ec on the temperature, and concentration profiles, respectively. It is seen that, the temperature increases by increasing of Eckert number Ec, but the concentration decreases by increasing of Eckert number Ec.And, we have led to Eckert number Ec effects on the nanoparticles the same effect of Eckert number Ec on the concentration.

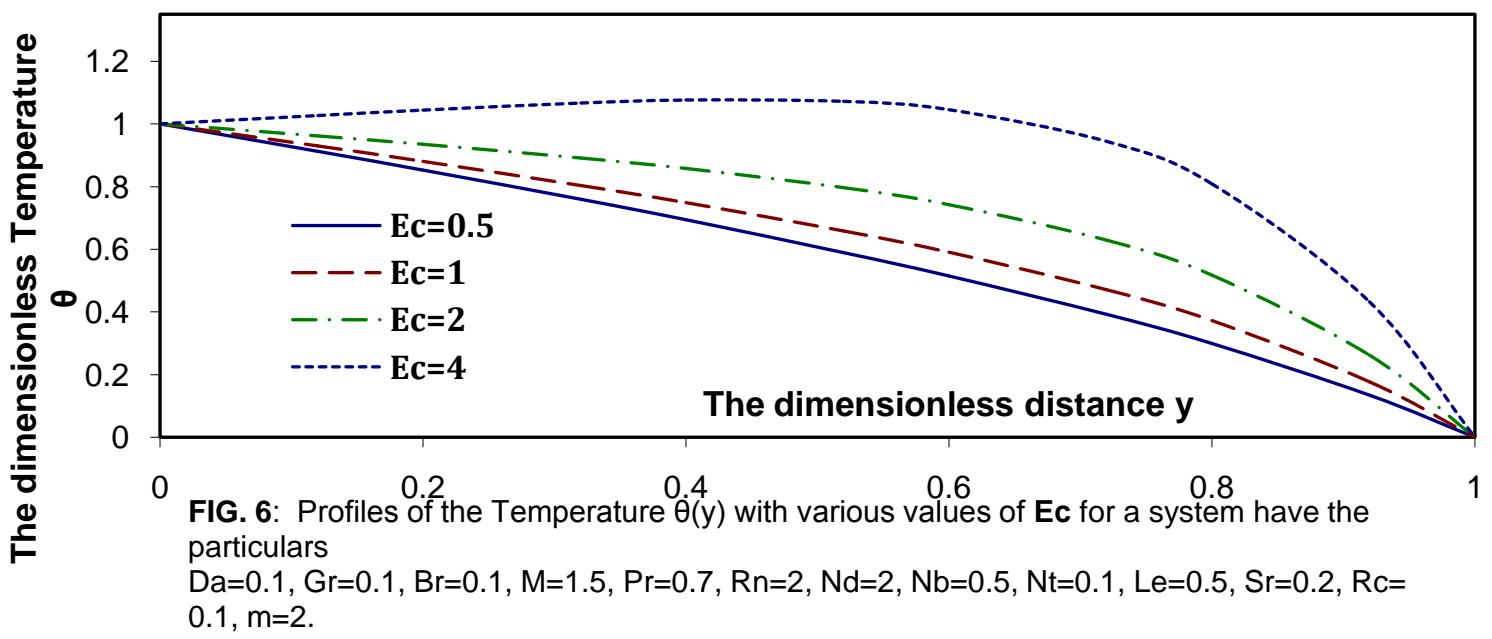




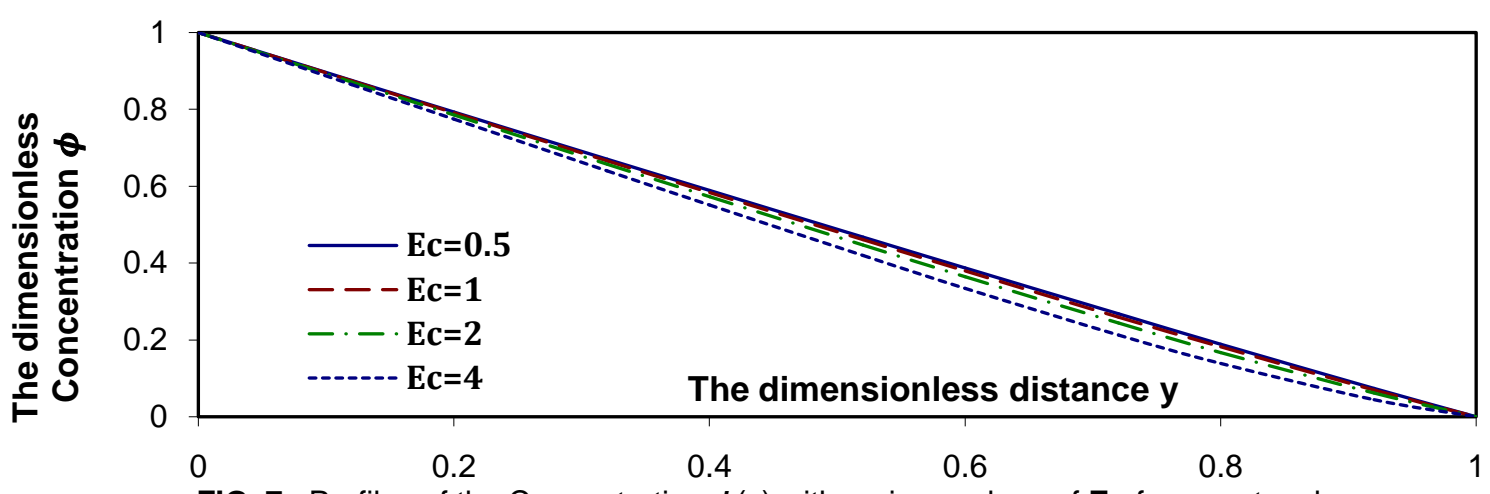

FIG. 7: Profiles of the Concentration $\phi(y)$ with various values of Ec for a system have the particulars

$\mathrm{Da}=0.1, \mathrm{Gr}=0.1, \mathrm{Br}=0.1, \mathrm{M}=1.5, \mathrm{Pr}=0.7, \mathrm{Rn}=2, \mathrm{Nd}=2, \mathrm{Nb}=0.5, \mathrm{Nt}=0.1, \mathrm{Le}=0.5, \mathrm{Sr}=0.2, \mathrm{R}$ $\mathrm{C}=0.1, \mathrm{~m}=2$.

Figure (8) illustrates the effect of Dufour number Nd on the nanoparticles profiles. It is seen that, the nanoparticles decreases by increasing of Dufour number Nd. And, we have led to Dufournumber Nd effects on the concentration the same effect of Dufour number $\mathrm{Nd}$ on the nanoparticles, and the opposite effect on the temperature.

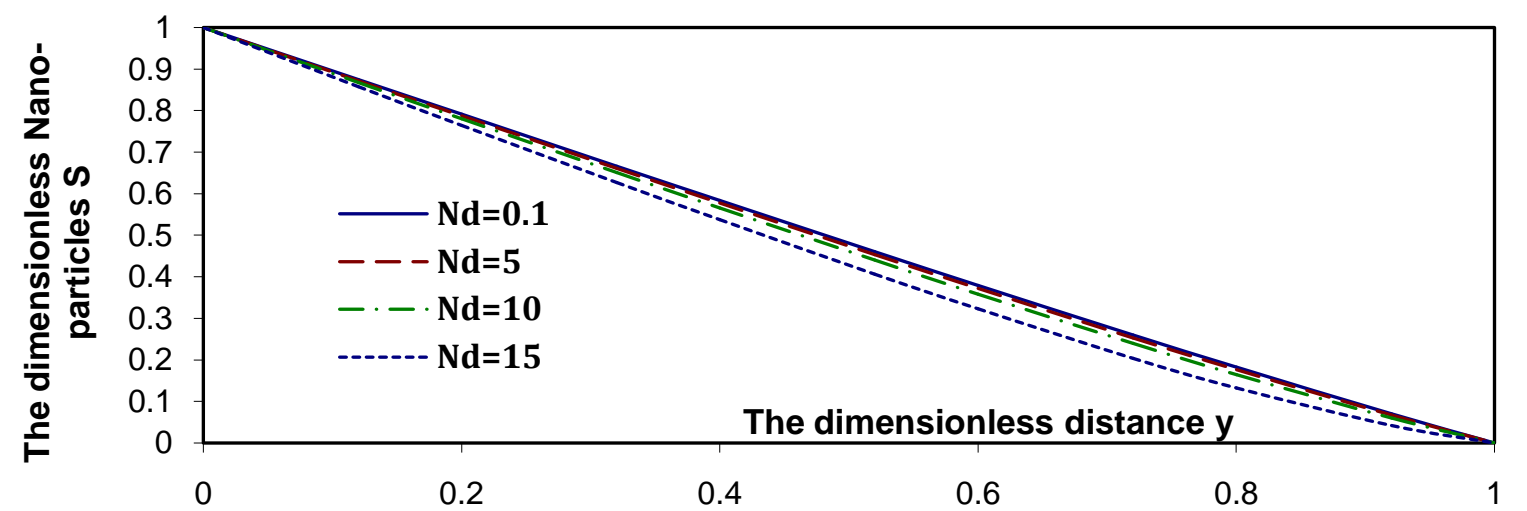

FIG. 8: Profiles of the Nano-particles $\mathrm{S}(\mathrm{y})$ with various values of $\mathrm{Nd}$ for a system have the particulars

$\mathrm{Da}=0.1, \mathrm{Gr}=0.1, \mathrm{Br}=0.1, \mathrm{M}=1.5, \mathrm{Pr}=0.7, \mathrm{Rn}=2, \mathrm{Ec}=0.5, \mathrm{Nb}=0.5, \mathrm{Nt}=0.1, \mathrm{Le}=0.5, \mathrm{Sr}=0.2$,

$\mathrm{Rc}=0.1, \mathrm{~m}=2$.

Figure (9) obtains the effect of Brownian motion parameter $\mathrm{Nb}$ on the concentration profiles. It is clear that, the concentration decreases by increasing of Brownian motion parameter $\mathrm{Nb}$. And, we have led to Brownian motion parameter $\mathrm{Nb}$ effects on the temperature and the nanoparticles the same effect of Brownian motion parameter $\mathrm{Nb}$ on the concentration.

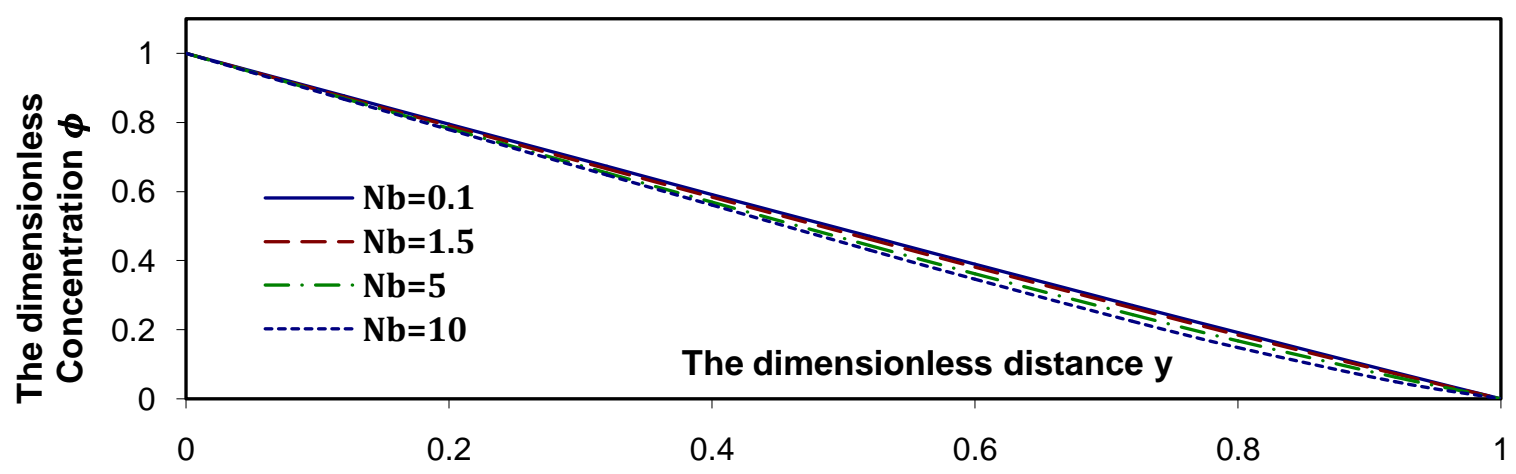

FIG. 9: Profiles of the Concentration $\phi(\mathrm{y})$ with various values of $\mathbf{N b}$ for a system have the particulars

$\mathrm{Da}=0.1, \mathrm{Gr}=0.1, \mathrm{Br}=0.1, \mathrm{M}=1.5, \mathrm{Pr}=0.7, \mathrm{Rn}=2, \mathrm{Ec}=0.5, \mathrm{Nd}=2, \mathrm{Nt}=0.1, \mathrm{Le}=0.5, \mathrm{Sr}=0.2, \mathrm{Rc}$ $=0.1, \mathrm{~m}=2$.

Figure $(10,11)$ shows the effect of Thermophoresis parameter $\mathrm{Nt}$ on the temperature and the 
Peristaltic Transport of a Third-Order Nano-Fluid in a Circular Cylindrical Tube with Radiation and Chemical Reaction

concentration, respectively. It is clear that, the temperature increases by increasing of Thermophoresis parameter $\mathrm{Nt}$, but the concentration decreases by increasing of Thermophoresis parameter Nt. And, we have led to Thermophoresis parameter Nt effects on the nanoparticles the same effect of Thermophoresis parameter Nt on the concentration.

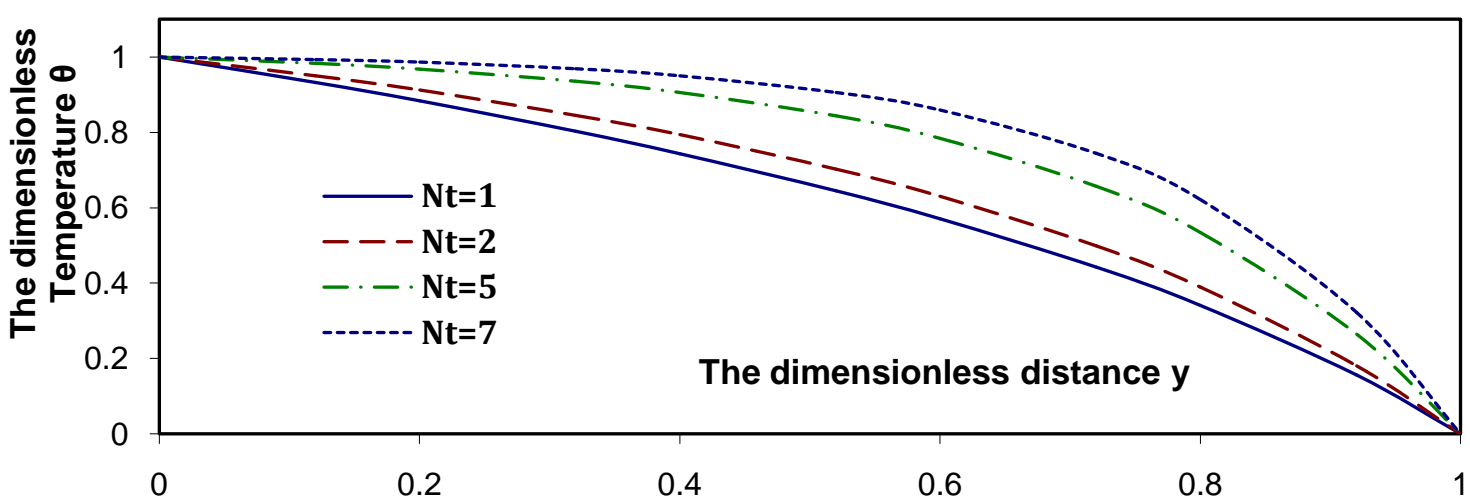

FIG. 10: Profiles of the Temperature $\theta(\mathrm{y})$ with various values of $\mathbf{N t}$ for a system have the particulars

$\mathrm{Da}=0.1, \mathrm{Gr}=0.1, \mathrm{Br}=0.1, \mathrm{M}=1.5, \mathrm{Pr}=0.7, \mathrm{Rn}=2, \mathrm{Ec}=0.5, \mathrm{Nd}=2, \mathrm{Nb}=0.5, \mathrm{Le}=0.5, \mathrm{Sr}=0.2, \mathrm{R}$ $\mathrm{c}=0.1, \mathrm{~m}=2$.

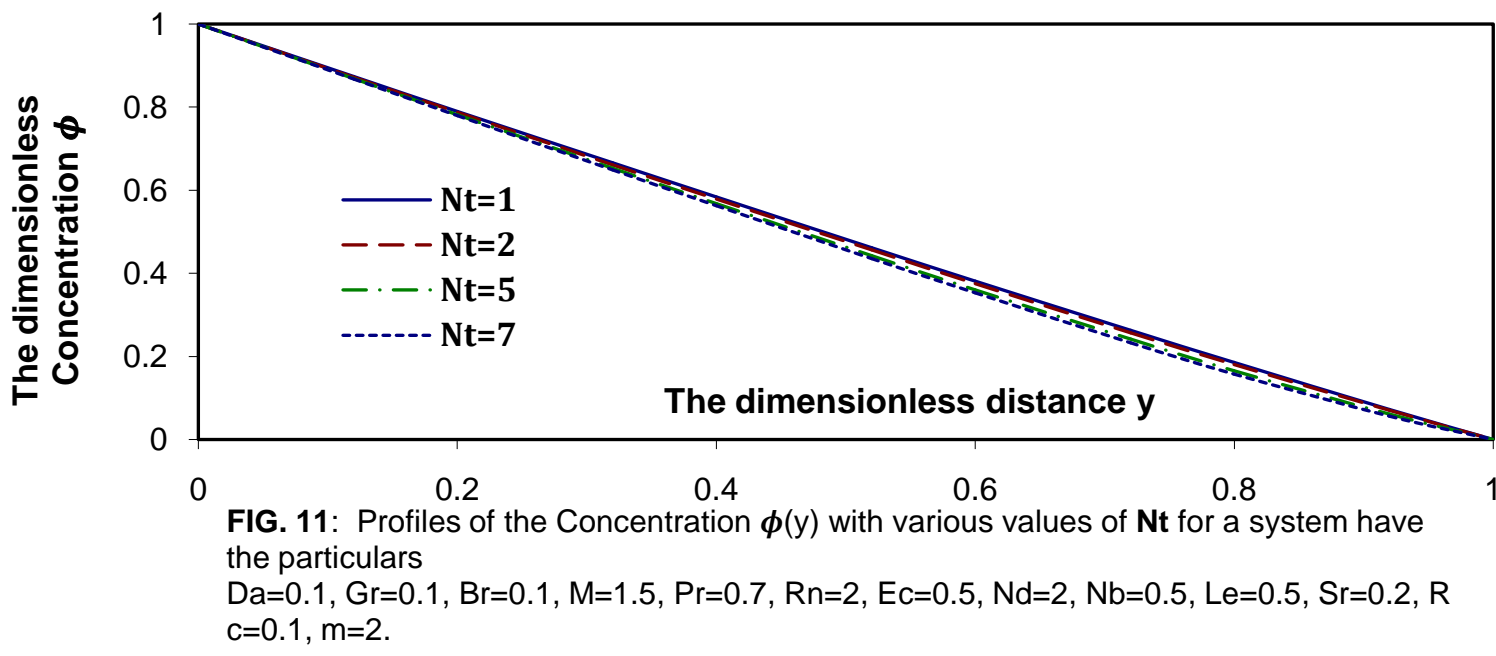

Figure (12) shows the effect of Prandtl number $\operatorname{Pr}$ on the nanoparticles. It is seen that, the nanoparticles decreases by increasing of Prandtl number Pr. And, we have led to Prandtl number Pr effects on the concentration the same effect of Prandtl number Pr on the nanoparticles, and the opposite effect on the temperature.

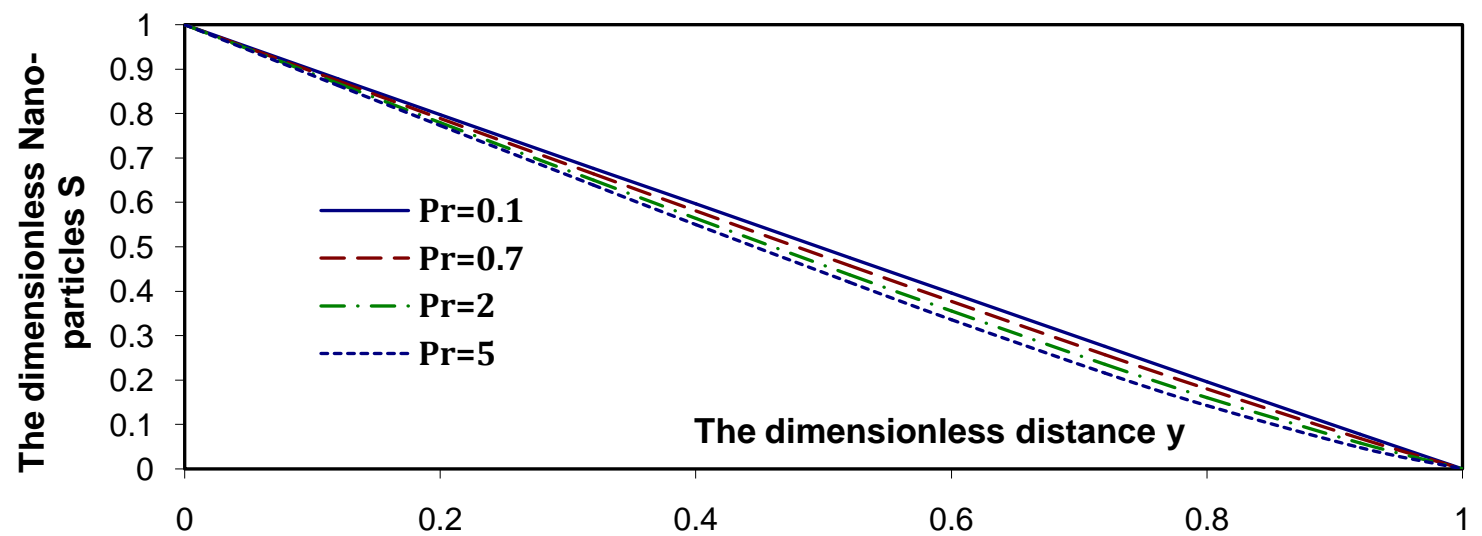

FIG. 12: Profiles of the Nano-particles $\mathbf{S}(\mathrm{y})$ with various values of $\operatorname{Pr}$ for a system have the particulars $\mathrm{Da}=0.1, \mathrm{Gr}=0.1, \mathrm{Br}=0.1, \mathrm{M}=1.5, \mathrm{Nt}=0.1, \mathrm{Rn}=2, \mathrm{Ec}=0.5, \mathrm{Nd}=2, \mathrm{Nb}=0.5, \mathrm{Le}=0.5, \mathrm{Sr}=0.2, \mathrm{R}$ $\mathrm{c}=0.1, \mathrm{~m}=2$. 
Figure $(13,14)$ illustrate the effect of radiation parameter Rnon the temperature and nanoparticles, respectively. It is shown that, the temperature increases by increasing of radiation parameter Rn, but the nanoparticles decreases by increasing of radiation parameter Rn. And, we have led to radiation parameter $\mathrm{Rn}$ effects on the concentration the same effect of radiation parameter $\mathrm{Rn}$ on the nanoparticles.

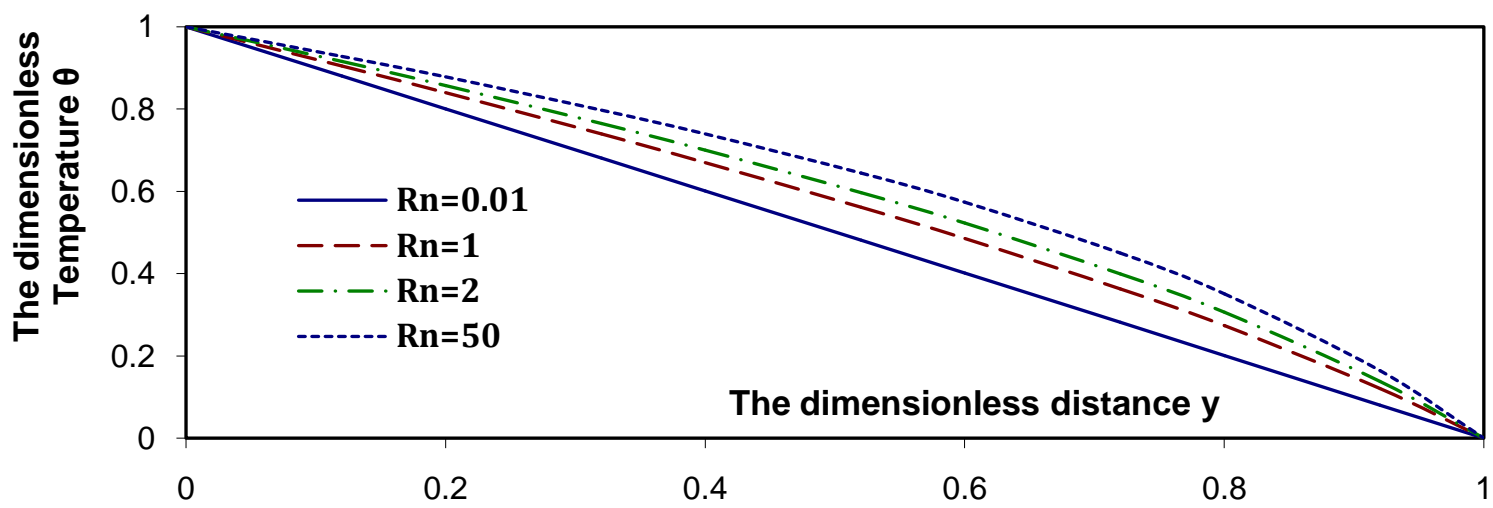

FIG. 13: Profiles of the Temperature $\theta(y)$ with various values of $\mathbf{R n}$ for a system have the particulars

$\mathrm{Da}=0.1, \mathrm{Gr}=0.1, \mathrm{Br}=0.1, \mathrm{M}=1.5, \mathrm{Nt}=0.1, \mathrm{Pr}=0.7, \mathrm{Ec}=0.5, \mathrm{Nd}=2, \mathrm{Nb}=0.5, \mathrm{Le}=0.5, \mathrm{Sr}=0.2$, $\mathrm{Rc}=0.1, \mathrm{~m}=2$.

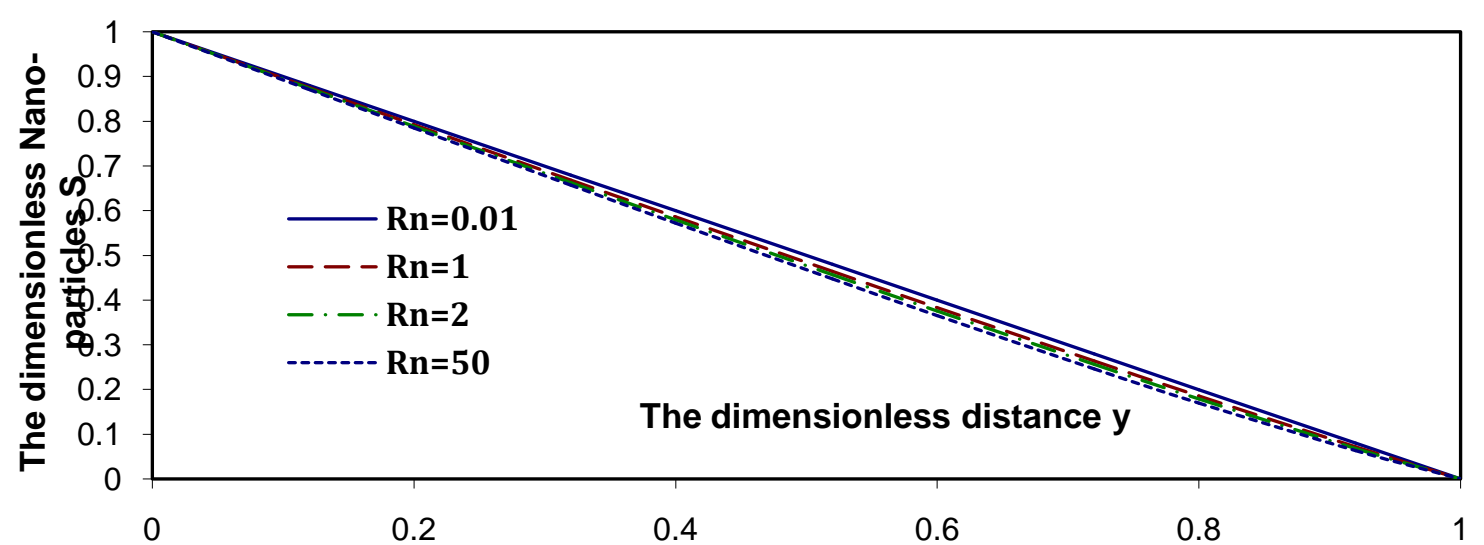

FIG. 14: Profiles of the Nano-particles $\mathbf{S}(\mathrm{y})$ with various values of $\mathbf{R n}$ for a system have the particulars

$\mathrm{Da}=0.1, \mathrm{Gr}=0.1, \mathrm{Br}=0.1, \mathrm{M}=1.5, \mathrm{Nt}=0.1, \mathrm{Pr}=0.7, \mathrm{Ec}=0.5, \mathrm{Nd}=2, \mathrm{Nb}=0.5, \mathrm{Le}=0.5, \mathrm{Sr}=0.2$,

$\mathrm{Rc}=0.1, \mathrm{~m}=2$.

Figure (15) shows the effect of Lewis number Le on the concentration. It is clear that, the concentration decreases by increasing of Lewis number Le. And, we have led to Lewis number Le effects on the nanoparticles the same effect of Lewis number Le on the concentration, and the opposite effect on the temperature.

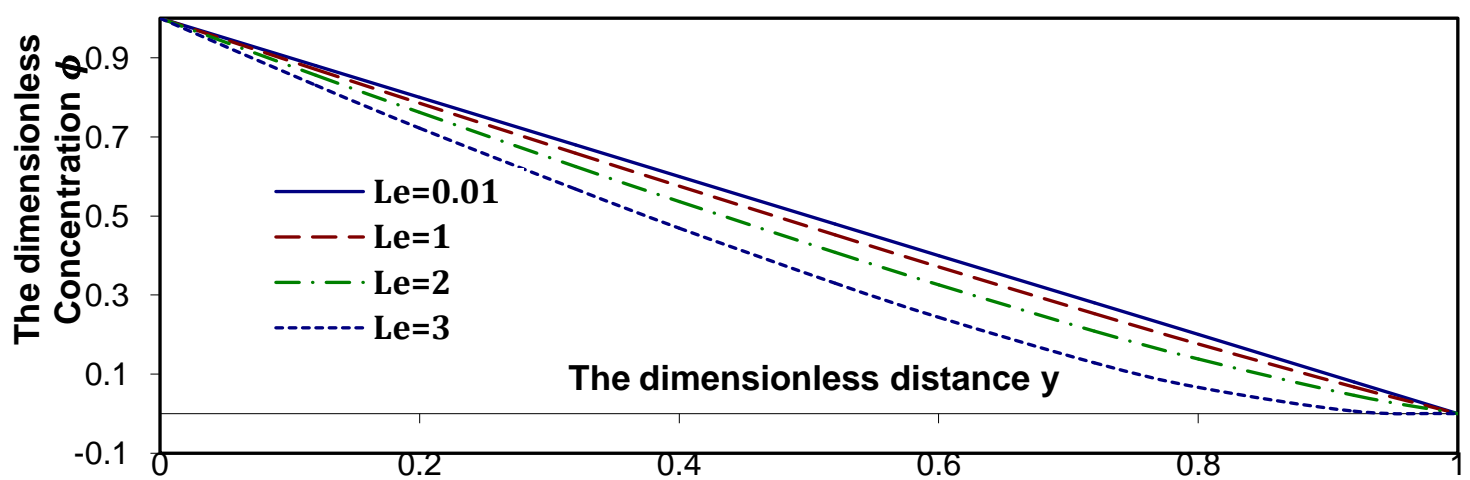

FIG. 15: Profiles of the Concentration $\phi(y)$ with various values of Le for a system have the particulars

$\mathrm{Da}=0.1, \mathrm{Gr}=0.1, \mathrm{Br}=0.1, \mathrm{M}=1.5, \mathrm{Nt}=0.1, \mathrm{Pr}=0.7, \mathrm{Ec}=0.5, \mathrm{Nd}=2, \mathrm{Nb}=0.5, \mathrm{Rn}=2, \mathrm{Sr}=0.2, \mathrm{R}$ $\mathrm{c}=0.1, \mathrm{~m}=2$. 
Figure (16) shows the effect of Sort number $\mathrm{Sr}$ on the nanoparticles. It is seen that, the nanoparticles decreases by increasing of Sort number Sr. And, we have led to Sort number Sr effects on the concentration the same effect Sort number Sr on the nanoparticles, and the opposite effect on the temperature.

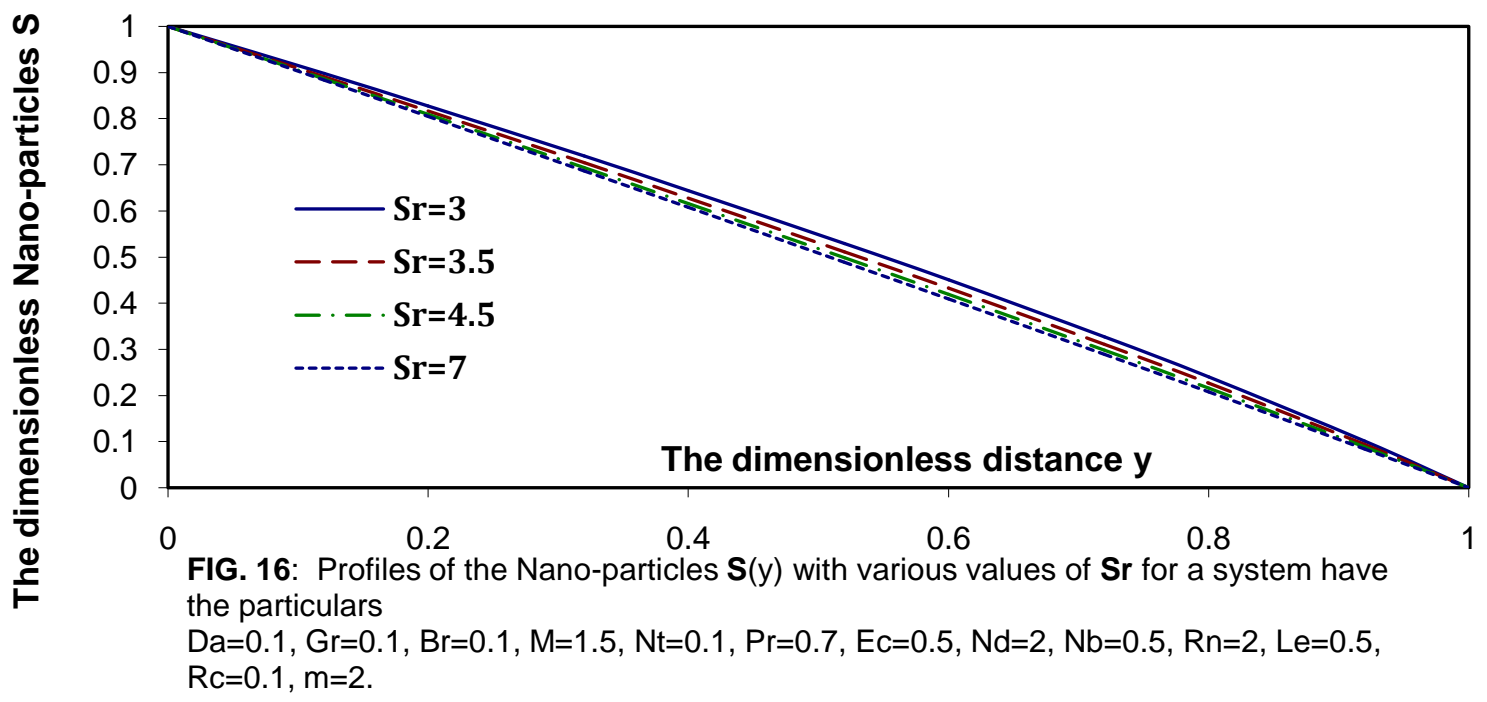

Figure (17) shows the effect of Chemical reaction parameter Rc on the concentration. It is clear that, the concentration decreases by increasing of Chemical reaction parameter Rc. And, we have led to Chemical reaction parameter Rc effects on the nanoparticles the same effect of Chemical reaction parameter Rc on the concentration, and the opposite effect on the temperature.

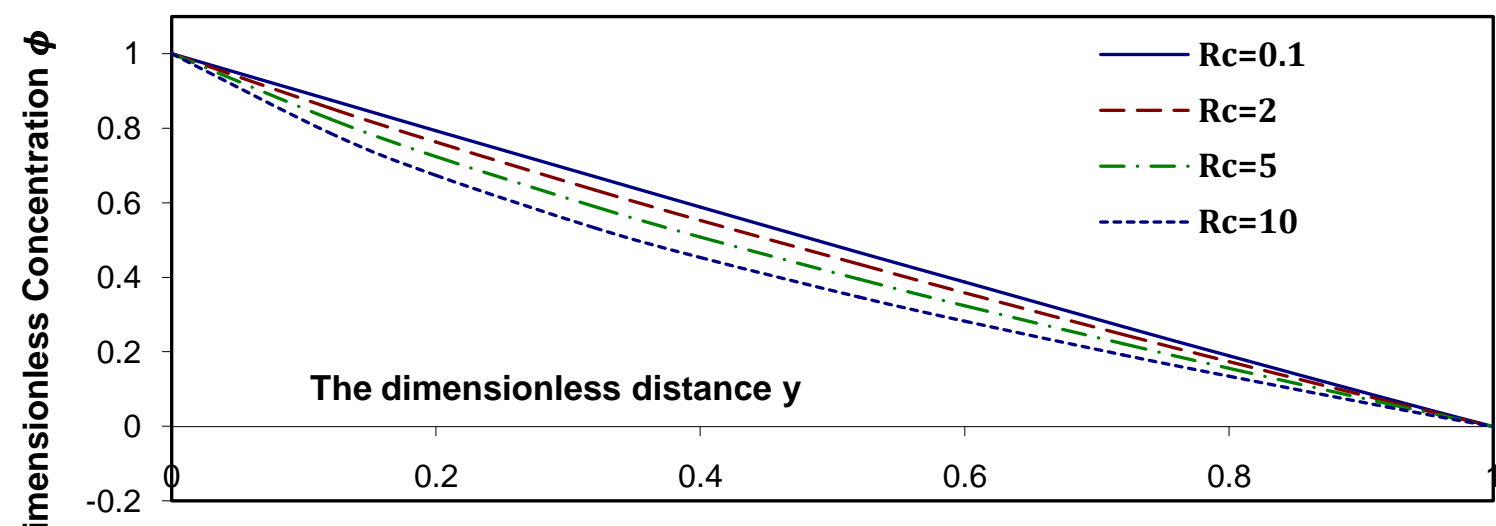

FIG. 17: Profiles of the Concentration $\phi(y)$ with various values of $\mathbf{R} \mathbf{c}$ for a system have the particulars $\mathrm{Da}=0.1, \mathrm{Gr}=0.1, \mathrm{Br}=0.1, \mathrm{M}=1.5, \mathrm{Nt}=0.1, \mathrm{Pr}=0.7, \mathrm{Ec}=0.5, \mathrm{Nd}=2, \mathrm{Nb}=0.5, \mathrm{Rn}=2, \mathrm{Le}=0.5, \mathrm{~S}$ $\mathrm{r}=0.2, \mathrm{~m}=2$.

\section{Conclusion}

In this work, we have studied a magneto-hydrodynamic third-order Nano-fluid with heat and mass transfer in a circular cylindrical tube having two walls that are transversely displaced by an infinite, harmonic traveling wave of large wave length. The governing boundary value problem was solved numerically by an Explicit Finite-Difference method. We concentrated our work on obtaining the stream function, the velocity, the temperature, the concentration, and the nanoparticlesdistributions which are illustrated graphically at different values of the parameters of the problem. Global error estimation is also obtained using Zadunaisky technique. We used 26 points to find the interpolating polynomial of degree 25 in interval $[0,1]$ and the results are shown in (tab.1 ). We notice that, the error in ( tab.1) is good enough to justify the use of resulting numerical values. 


\section{REFERENCES}

[1] HayatT. and AliN., Peristaltically induced motion of a MHD third grade fluid in a deformable tube, Physica A. 370, 225, (2006).

[2] Latham T. W., Fluids Motions in a Peristaltic Pump, Thesis, M. I. T., Cambridge, (1966).

[3] HarounM. H., Effect of Deborah Number and Phase Difference on Peristaltic transport of a Third-Order Fluid in an Asymmetric Channel, Communication in Nonlinear Science and Numerical Simulation.12, 1464, (2007).

[4] EldabeN. T. M., El-SayedM. F., GhalyA. Y. and SayedH. M., Peristaltically Induced Transport of a MHD Biviscosity Fluid in a Non-Uniform Tube, Physica A. 383, 253, (2007).

[5] HayatT., AfsarA., KhanM.and AsgharS.,Peristaltic transport of a third order fluid under the effect of a magnetic field, Computers and Mathematics with Applications. 53, 1074, (2007).

[6] EllahiR., RazaM. and VafaiK.,Series solutions of non-Newtonian nanofluids with Reynolds' model and Vogel's model by means of the homotopy analysis method, Mathematical and Computer Modelling. 55, 1876, (2012).

[7] ChoiS. U., Enhancing thermal conductivity of fluids with nanoparticle, ASME J. Develop.

[8] Appl. Non-Newtonian Flows. 66, 99, (1995).

[9] FarooqU., HayatT., AlsaediA.and LiaoShijun, Heat and mass transfer of two-layer flows of third-grade nano-fluids in a vertical channel, Applied Mathematics and Computation. 242, 528, (2014).

[10] TzirtzilakisE. E., A mathematical model for blood flow in magnetic field, Physics of Fluids. 17,077103, (2005).

[11] BakierA. Y., Thermal radiation effect on mixed convection from vertical surface in saturated porous media, International Journal of Communication of Heat and Mass Transfer.28, 119, (2001).

[12] DamsehR. A., Magnetohydrodynamics-mixed convection from radiate vertical isothermal surface embedded in a saturated porous media, Journal of Applied Mechanics. 73, 54, (2006).

[13] HossainM. A. and TakharH. S., Radiation effect on mixed convection along a vertical plate with uniform surface temperature, Heat and Mass Transfer. 31, 243, (1996).

[14] ZahmatkeshI., Influence of thermal radiation on free convection inside a porous enclosure, Emirates Journal for Engineering Research. 12, 47, (2007).

[15] SheikholeslamiMohsen, DomiriGanjiDavood, YounusJavedM. and EllahiR., Effect of thermal radiation on magnetohydrodynamicsnanofluids flow and heat transfer by means of two phase model, Journal of Magnetism and Magnetic Materials. 374, 36, (2015).

[16] HinaS., HayatT., AsgharS. and HendiAwatif A., Influence of compliant walls on peristaltic motion with heat/mass transfer and chemical reaction, International Journal of Heat and Mass Transfer. 55, 3386, (2012).

[17] VajraveluK., PrasadK. V.and AbbasbandyS., Convection transport of nanoparticle in multi-layer fluid flow, Appl. Math. Mech. Eng. Ed.34(2), 177 (2013).

[18] FarooqU., HayatT., AlsaediA. and LiaoShijun, Heat and mass transfer of two-layer flows of third-grade nano-fluids in a vertical channel, Appl. Math. Comp. 242, 528, (2014).

[19] Abou-zeidMohamed Y., ShaabanAbeer A. and AlnourMuneer Y., Numerical treatment and global error estimation of natural convective effects on gliding motion of bacteria on a powerlaw nanoslime through a non-Darcy porous medium, J. Porous Media. 18(11), 1091,(2015).

[20] El-dabeNabil T.M., ShaabanAbeer A., Abou-zeidMohamed Y.and AliHemat A., MHD nonNewtonian nanofluid flow over a stretching sheet with thermal diffusion and diffusion thermo effects through a non-Darcy porous medium with radiation and chemical reaction, J. Compu. Theor. Nanoscience. 12(11), 5363, ( 2015).

[21] ShaabanA. A. and Abou-zeidM. Y., Effects of Heat and Mass Transfer on MHD Peristaltic Flow of a Non-Newtonian Fluid through a Porous Medium between Two Coaxial Cylinders, Mathematical Problems in Engineering, 2013, 1, (2013).

[22] ZadunaiskyP. E., On the Estimation of Errors Propagated in the Numerical Integration of Ordinary Differential Equations, Numerical Mathematics.27,21, (1976). 
[23] FosdickR. L. and RajagopalK. R., Thermodynamics and stability of fluids of third-grade, Proc. Roy. Soc. London A. 339, 351, (1980).

[24] DunnJ. E. and RajagopalK. R., Fluids of differential type-critical-review and thermos dynamic analysis, Int. J. Eng. Sci. 33, 689, (1995).

[25] EldabeN. T. M., HassanA. A.and MohamedM. A. A., Effect of couple stresses on the MHD of a non-Newtonian unsteady flow between two parallel porous plates, Z. Naturforsch. 58 a, 204, (2003).

[26] EldabeN. T. M.and MohamedM. A. A., Heat and mass transfer in Hydromagnetic flow of the non-Newtonian fluid with heat source over an accelerating surface through a porous medium, Chaos, Solitons and Fractals. 13, 907, (2002).

[27] C. P. Arora, Heat and Mass transfer, Khanna Publishers Delhi, (1997).

[28] RaptisA., Flow of a micropolar fluid past a continuously moving plate by presence of radiation, Int. J. Heat and Mass Transfer. 41, 2865, (1998).

[29] ShapiroA. H., JaffrinM. Y. and WeinbergS. L., Peristaltic pumping with long wavelengths at low Reynolds number, J. Fluid Mech. 37, 799, (1969). 